Full length article

\title{
Alignment of collagen fiber in knitted silk scaffold for functional massive rotator cuff repair
}

\author{
Zefeng Zheng ${ }^{\mathrm{a}, \mathrm{b}, \mathrm{j}, 1}$, Jisheng Ran ${ }^{\mathrm{b}, \mathrm{i}, 1}$, Weishan Chen ${ }^{\mathrm{b}, \mathrm{i}, 1}$, Yejun $\mathrm{Hu}^{\mathrm{a}, \mathrm{b}}$, Ting Zhu ${ }^{\mathrm{b}}$, Xiao Chen ${ }^{\mathrm{a}, \mathrm{ch}}{ }^{\mathrm{h}}$, Zi Yin ${ }^{\mathrm{a}, \mathrm{c}}$, \\ Boon Chin Heng ${ }^{\mathrm{d}}$, Gang Feng ${ }^{\mathrm{b}}$, Huihui Le ${ }^{\mathrm{b}, \mathrm{i}}$, Chenqi Tang ${ }^{\mathrm{b}, \mathrm{i}}$, Jiayun Huang ${ }^{\mathrm{b}, \mathrm{i}}$, Yangwu Chen ${ }^{\mathrm{b}, \mathrm{i}}$, Yiting Zhou ${ }^{\mathrm{e}, \mathrm{f}}$, \\ Pioletti Dominique ${ }^{g}$, Weiliang Shen ${ }^{\mathrm{a}, \mathrm{b}, \mathrm{c}, \mathrm{e}, \mathrm{g}, \mathrm{h}, \mathrm{i}, \mathrm{i}, *}$, Hong-wei Ouyang ${ }^{\mathrm{a}, \mathrm{c}, \mathrm{e}, \mathrm{h}}$
}

${ }^{a}$ Dr. Li Dak Sum E' Yip Yio Chin Center for Stem Cell and Regenerative Medicine, Zhejiang University, Zhejiang 310000, China

${ }^{\mathrm{b}}$ Department of Orthopedic Surgery, 2nd Affiliated Hospital, School of Medicine, Zhejiang University, Zhejiang 310009, China

${ }^{\mathrm{c}}$ Key Laboratory of Tissue Engineering and Regenerative Medicine of Zhejiang Province, School of Medicine, Zhejiang University, Zhejiang 310009, China

${ }^{\mathrm{d}}$ Faculty of Dentistry, The University of Hong Kong, Pokfulam, Hong Kong

e Department of Sports Medicine, School of Medicine, Zhejiang University, Zhejiang 310000, China

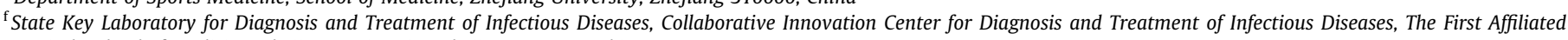
Hospital, School of Medicine, Zhejiang University, Zhejiang 310003, China

${ }^{\mathrm{g}}$ Laboratory of Biomechanical Orthopedics, EPFL, Lausanne, Switzerland

${ }^{\text {h } C h i n a ~ O r t h o p a e d i c ~ R e g e n e r a t i v e ~ M e d i c i n e ~(C O R M e d), ~ H a n g z h o u, ~ C h i n a ~}$

${ }^{i}$ Department of Orthopedics Research Institute of Zhejiang University, Zhejiang, China

${ }^{j}$ Department of Orthopedic Surgery, The Children's Hospital, School of Medicine, Zhejiang University, Zhejiang 310052, China

\section{A R T I C L E I N F O}

\section{Article history:}

Received 30 August 2016

Received in revised form 11 January 2017

Accepted 12 January 2017

Available online 16 January 2017

\section{Keywords:}

Macroporous three-dimensional aligned

collagen/silk scaffold

Rotator cuff

Tissue engineering

Regeneration

\begin{abstract}
A B S T R A C T
Rotator cuff tear is one of the most common types of shoulder injuries, often resulting in pain and physical debilitation. Allogeneic tendon-derived decellularized matrices do not have appropriate pore size and porosity to facilitate cell infiltration, while commercially-available synthetic scaffolds are often inadequate at inducing tenogenic differentiation. The aim of this study is to develop an advanced 3D aligned collagen/silk scaffold (ACS) and investigate its efficacy in a rabbit massive rotator cuff tear model. ACS has similar 3D alignment of collagen fibers as natural tendon with superior mechanical characteristics. Based on ectopic transplantation studies, the optimal collagen concentration $(10 \mathrm{mg} / \mathrm{ml})$, pore diameter $(108.43 \pm 7.25 \mu \mathrm{m})$ and porosity $(97.94 \pm 0.08 \%)$ required for sustaining a stable macro-structure conducive for cellular infiltration was determined. Within in vitro culture, tendon stem/progenitor cells (TSPCs) displayed spindle-shaped morphology, and were well-aligned on ACS as early as $24 \mathrm{~h}$. TSPCs formed intercellular contacts and deposited extracellular matrix after 7 days. With the in vivo rotator cuff repair model, the regenerative tendon of the ACS group displayed more conspicuous native microstructures with larger diameter collagen fibrils $(48.72 \pm 3.75$ vs. $44.26 \pm 5.03 \mathrm{~nm}$ ) that had better alignment and mechanical properties $(139.85 \pm 49.36$ vs. $99.09 \pm 33.98 \mathrm{~N})$ at 12 weeks post-implantation. In conclusion, these findings demonstrate the positive efficacy of the macroporous 3D aligned scaffold in facilitating rotator cuff tendon regeneration, and its practical applications for rotator cuff tendon tissue engineering.
\end{abstract}

\section{Statement of Significance}

Massive rotator cuff tear is one of the most common shoulder injuries, and poses a formidable clinical challenge to the orthopedic surgeon. Tissue engineering of tendon can potentially overcome the problem. However, more efficacious scaffolds with good biocompatibility, appropriate pore size, favorable inductivity and sufficient mechanical strength for repairing massive rotator cuff tendon injuries need to be developed. In this study, we developed a novel macroporous 3D aligned collagen/silk scaffold, and demonstrated that this novel scaffold enhanced the efficacy of rotator cuff tendon regeneration by inducing aligned supracellular structures similar to natural tendon, which in turn enhanced

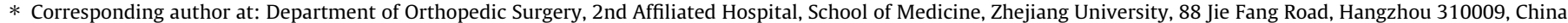
E-mail address: shenweiliang365@163.com (W. Shen).

1 These co-authors contributed equally to the work.
} 
cellular infiltration and tenogenic differentiation of stem/progenitor cells from both the tendon itself and surrounding tissues. Hence, it can potentially be a clinically useful application for tendon tissue engineering.

(c) 2017 Acta Materialia Inc. Published by Elsevier Ltd. All rights reserved.

\section{Introduction}

Rotator cuff tendon injury is one of the most common types of injuries encountered during overhead activities and sports, often resulting in much pain and physical debilitation $[1,2]$. About $10 \%$ to $40 \%$ of all rotator cuff tears are massive rotator cuff tears that present a formidable clinical challenge to the orthopedic surgeon, and which often require implantable devices for therapy [3]. Tissue grafts, particularly allograft is the current gold standard in clinical practice. However allogeneic tendon-derived decellularized matrices do not have appropriate pore size and porosity to facilitate cell infiltration [4]. Commercial products based on the decellularized matrix of other tissues, such as Restore ${ }^{\circledR}$, Bio-Blanket ${ }^{\circledR}$ and TissueMend $^{\circledR}$, are also used in rotator cuff repair [5]. However, their effects on tenogenic differentiation are usually weaker than tendon-derived decellularized matrices [6]. In addition, synthetic scaffolds have the drawback of poor cell adhesion, and can induce chronic inflammation $[7,8]$. Hence, more efficacious scaffolds with good biocompatibility, appropriate pore size, favorable inductivity and sufficient mechanical strength for facilitating repair of massive rotator cuff tendon injuries need to be developed.

Tissue engineering of tendon can potentially overcome some of these deficiencies and promote the healing of tendon injury $[9,10]$. One of the key components of tendon tissue engineering is the scaffold. Collagen has many advantageous properties such as ubiquity, low antigenicity, biocompatibility and biodegradability, and has been widely utilized as a scaffold biomaterial [11]. In particular, type I collagen is the major component of tendon extracellular matrix, and has been extensively utilized in tendon tissue engineering [12]. Nevertheless, regenerated tendon tissue within sponge collagen scaffold is disordered and irregular, which can have a negative impact on the biomechanical function of the regenerated tendon [13]. Hence, it is imperative to further improve the collagen scaffold utilized for tendon repair.

Recent studies showed that aligned scaffolds can exert a profound influence on cell morphology and tendon-related gene expression, resulting in enhancement of tenogenic differentiation both in vitro and in vivo with animal injury models [14-17]. However, aligned scaffolds fabricated by the electrospinning procedure are nanoporous, usually having low porosity with small pores between fibers. These pores are smaller than the typical cell size. As a result, cells can only grow on the top of the scaffold, leading to low cell infiltration rates being observed in vivo [18]. Although much effort has been devoted to increasing the porosity and pore size $[19,20]$, a robust and implantable aligned scaffold has not yet been developed, due mainly to the reduction of mechanical strength with increasing pore size and porosity.

Because pure collagen scaffold exhibit poor mechanical properties in vivo, it is necessary to combine it with a material that possesses good mechanical strength [13]. Silk is a FDA -approved material, which has been widely utilized in tissue engineering due to its good biocompatibility and mechanical strength [21]. In particular, knitted silk scaffold can meet many of the requirements for tendon tissue engineering [22].

The aim of this study is to fabricate a macroporous 3D aligned collagen-silk scaffold with optimal pore size (several hundred micrometers), adequate porosity (higher than 90\%) [20], favorable inductivity (enhancing tenogenic differentiation) and sufficient mechanical strength (no tear after implantation in vivo) [13], and to investigate its effect on tendon remodeling during functional shoulder repair in a rabbit massive rotator cuff defect model.

\section{Materials and methods}

\subsection{Scaffold fabrication}

Raw Bombyx mori silk fibers were purchased from Zhejiang Cathaya International Co. Ltd. The knitted silk was fabricated as described previously [13]. Briefly, the knitted scaffold was fabricated using 12 yarns (1 filament/yarn) of silk fibers on a knitting machine. Plain knitted silk scaffolds were fabricated with 21 stitches per centimeter. The knitted scaffolds were then processed to extract sericin, by rinsing three times in an aqueous solution containing $0.02 \mathrm{M} \mathrm{Na}_{2} \mathrm{CO}_{3}$ at $90{ }^{\circ} \mathrm{C}$ and $100{ }^{\circ} \mathrm{C}$ for $60 \mathrm{~min}$. The pore size was approximately $1 \times 1 \mathrm{~mm}$.

Insoluble type I collagen was derived from pig Achilles' tendon using neutral salt and dilute acid extractions with enzyme treatment $[13,23]$. Type III collagen was removed by centrifugation after salt fractionation, and type I collagen was drawn off the lower portion. The macroporous 3D aligned collagen scaffold was produced by unidirectional freezing technology in a horizontal direction. The collagen was held in perspex molds $(20 \times 20 \times 5 \mathrm{~mm}$; type I, $\mathrm{pH} 3.2,6 / 10 / 18 \mathrm{mg} / \mathrm{ml}$ ) with $\mathrm{L}$ shaped aluminum rods (including two heads: long head and short head). Freezing temperature was created by liquid nitrogen (LN2) through the short head of the aluminum rods, and transmitted along the aluminum rod. A temperature gradient was created, and the collagen froze from the long head of the aluminum rod, and became aligned. To produce collagen sponge scaffolds, the collagen was held in perspex molds $(20 \times 20 \times 5 \mathrm{~mm}$; type I, pH $3.2,6 / 10 / 18 \mathrm{mg} / \mathrm{ml})$ without aluminum rods, and frozen at $-80^{\circ} \mathrm{C}$ for $12 \mathrm{~h}$. To fabricate macroporous 3D aligned collagen/silk scaffold, the collagen was initially held in perspex molds $(20 \times 20 \times 2.5 \mathrm{~mm}$; type $\mathrm{I}$, pH 3.2 , $6 / 10 / 18 \mathrm{mg} / \mathrm{ml}$ ). Then, the knitted silk was placed flat on the top of the collagen solution. Finally, the rest of the collagen $(20 \times 20 \times 2.5 \mathrm{~mm}$; type $\mathrm{I}, \mathrm{pH} 3.2,6 / 10 / 18 \mathrm{mg} / \mathrm{ml})$ was added to the knitted silk before freezing. The scaffolds were freeze-dried under vacuum (Heto PowerDry LL1500) for 48 h to remove water, and were then crosslinked by dehydrothermal treatment $\left(22^{\circ} \mathrm{C}\right.$ for $24 \mathrm{~h}, 110^{\circ} \mathrm{Cfor} 72 \mathrm{~h}$, and $65^{\circ} \mathrm{Cfor} 24 \mathrm{~h}$, at vacuum pressure $<10$ mbar) [13]. The structural morphology of the scaffolds (longitudinal section) was characterized by light microscopy and scanning electron microscopy (SEM), and the biomechanical properties of the scaffolds were evaluated by an Instron tension/compression system.

The porosity of the scaffold was calculated according to the following formula ( $\mathrm{n}=5$ for each group):

Porosity $(\%)=\left(1-V_{c} / V_{t}\right) \times 100 \%=(1-m / \rho) \times 100 \%$

where $V_{c}$ is the volume occupied by the collagen, $V_{t}$ is the total volume of the scaffold, $m$ is the mass of the scaffold, $\rho$ is the density of anhydrous collagen, assumed to be $1.3 \mathrm{~g} / \mathrm{ml}$ [24].

The pore diameter of scaffold was measured by image analysis software (Image-Pro Plus, Rockville, MD, USA). 


\subsection{Ectopic implantation}

To determine the optimal collagen concentration and porosity for the composite scaffold, three female rabbits weighing about $2.5 \mathrm{~kg}$ were subjected to ectopic implantation. The study protocol was approved by the Zhejiang University Animal Care and Use Committee (ZJU2010102003). The rabbits were initially subjected to general anesthesia by administration of chloral hydrate. A $20 \mathrm{~mm}$ incision was then made on the dorsum, and the collagen scaffold $(20 \times 20 \times 5 \mathrm{~mm})$ was sutured to the fascia in a p pocket by blunt dissection away from the incision. The macroporous 3D aligned collagen scaffold was sutured on the left side, while the collagen sponge scaffold was sutured on the right side. Each side had three scaffolds with different collagen concentrations $(6,10$ and $18 \mathrm{mg} / \mathrm{ml}$ ), and the incision was closed by non-resorbable suture. Two weeks after implantation, the animals were subjected to euthanasia, and the implanted tissues were harvested for hematoxylin and eosin (HE) staining.

\subsection{Cell seeding in scaffolds}

Rabbit tendon stem/progenitor cells (rTSPCs) were isolated as described previously [14]. Briefly, rotator cuff tendons of female rabbits (6 months old) were collected, washed in phosphatebuffered saline (PBS), and cut into $1-2 \mathrm{~mm}^{3}$ pieces. The tendon pieces were then digested with $0.25 \%(\mathrm{w} / \mathrm{v})$ collagenase (SigmaAldrich Inc., St. Louis, MO, USA) at $37^{\circ} \mathrm{C}$ overnight. Single cell suspensions were cultured in growth medium consisting of Dulbecco's modified Eagle's medium (DMEM, low glucose; Gibco-BRL Inc., Long Island, NY, USA) supplemented with $10 \%(\mathrm{v} / \mathrm{v})$ fetal bovine serum (FBS; Invitrogen Inc., Carlsbad, CA, USA) and $1 \%(\mathrm{v} / \mathrm{v})$ penicillin-streptomycin (Gibco-BRL Inc.). Fresh culture medium was replenished once every 3 days. The characterization of rTSPCs has been demonstrated in our previous study. The rTSPCs presented fibroblast-like morphology, and exhibited clonogenicity, self-renewal and multipotency [22].

Collagen/silk scaffolds $(10 \times 10 \times 5 \mathrm{~mm} ; 10 \mathrm{mg} / \mathrm{ml}$ collagen concentration) were treated with $75 \%$ ethanol $(\mathrm{v} / \mathrm{v})$ for $1 \mathrm{~h}$ and washed three times with sterile PBS, and were seeded with $2 \times 10^{5}$ rTSPCs in 24-well culture plates. Three samples from each group were harvested at $24 \mathrm{~h}$ for HE staining. Image analysis software (Image-Pro Plus, Rockville, MD, USA) was used to measure the average length and angle distribution of the cells. Cellular proliferation was quantified by the cell counting kit-8 (CCK8, Dojindo, Kumamoto, Japan) on day 1, 3, and 7 after seeding, according to the manufacturer's instructions, with absorbance readings being measured at $450 \mathrm{~nm}$. At 1, 3, and 7 days after seeding, the cell morphology and distribution were imaged with SEM. The cell-seeded scaffolds were immersed in $\mathrm{OsO}_{4}$ for $40 \mathrm{~min}$ and then washed three times in PBS. After dehydration in increasing concentrations of acetone (30-100\%,v/v), the samples were coated with gold, and then imaged under a Hitachi S-3000N scanning electron microscope (Hitachi Corp., Tokyo, Japan) at an accelerating voltage of $15 \mathrm{kV}$.

\subsection{Animal model}

Twenty female rabbits weighing about $2.5 \mathrm{~kg}$ were utilized. Treatment of animals was in accordance with standard guidelines approved by the Zhejiang University Ethics Committee. After anesthesia, the rabbits' rotator cuff tendons were exposed by releasing parts of the trapezius and deltoid muscles through a longitudinal incision over the shoulder. A portion of the rotator cuff tendon was fully removed to create a rectangular defect of approximately $10 \times 5 \mathrm{~mm}$ in area (about 50\% of the rotator cuff tendon). The left defects were implanted with macroporous 3D aligned collagen/silk scaffold $(20 \times 20 \times 2.5 \mathrm{~mm}$; type I, pH 3.2, $10 \mathrm{mg} / \mathrm{ml})$ using a nonresorbable suture (4-0 nylon), while the right defects were implanted with sponge collagen/silk scaffolds $(20 \times 20 \times 2.5 \mathrm{~mm}$; type I, pH 3.2, $10 \mathrm{mg} / \mathrm{ml}$ ). After surgery, the rabbits were allowed free cage activity with equal access to food and water, and monitored on a daily basis until they were culled. Five samples from each group were harvested to be cut from the middle into two segments at the 4,8 , and 12 weeks time-points, one for histological evaluation, and the other for gene expression analysis and quantitation of collagen content. Three randomly selected samples from the segments for gene expression analysis and quantitation of collagen content were imaged by transmission electron microscopy (TEM) at 8 and 12 weeks post-surgery. Another five samples containing the humerus head and rotator cuff tendon from each group were utilized for mechanical testing at 12 weeks postimplantation.

\subsection{Histological assessment}

Specimens were immediately fixed in $10 \%(\mathrm{w} / \mathrm{v})$ neutral buffered formalin, dehydrated through an increasing ethanol gradient, cleared, and embedded in paraffin blocks. Histological sections $(8 \mu \mathrm{m})$ were prepared using a microtome, and subsequently deparaffinized with xylene, hydrated using decreasing concentrations of ethanol, and then subjected to HE staining. In addition, Masson trichrome staining was performed according to standard procedures to examine the general appearance of the collagen fibers. Polarizing microscopy was employed to detect mature collagen fibrils. Histological scoring was performed by three people using a blinded semi-quantitative scoring system based on six parameters (fiber structure, fiber arrangement, rounding of nuclei, vascularity, inflammation, cell population) of HE staining. Every parameter was graded from 0 to 3 , with 0 being normal and 3 being maximally abnormal [15].

\subsection{RNA isolation and quantitative $P C R$}

Total tissue RNA ( $\mathrm{n}=5$ for each group) was isolated by lysis in TRIZOL (Invitrogen Inc., Carlsbad, CA, USA) followed by a onestep phenol chloroform-isoamyl alcohol extraction as described by the manufacturer's protocol. The expression levels of tendonrelated genes were assessed by quantitative PCR using a Brilliant SYBR Green QPCR Master Mix (TakaRa) with a Light Cycler apparatus (ABI 7900HT). The PCR cycling consisted of 40 cycles of amplification of the template DNA with primer annealing at $60^{\circ} \mathrm{C}$. The primer sequences used in this study are based on a previous study [25] and are listed in Table 1. Each quantitative PCR run was performed with at least three experimental replicates, and the relative expression level of each gene was calculated using the $2^{-\Delta \Delta \mathrm{Ct}}$ method.

Table 1

List of primer sequences utilized for real-time Polymerase Chain Reaction.

\begin{tabular}{|c|c|c|c|}
\hline Genes & $5^{\prime}-3^{\prime}$ & Primers & $\begin{array}{l}\text { Production } \\
\text { size (bp) }\end{array}$ \\
\hline \multirow[t]{2}{*}{ Collagen I } & Forward & GATGGCCTGAAGCTCAA & 412 \\
\hline & Reverse & GGTTTGTTGAAGAGGCTG & \\
\hline \multirow[t]{2}{*}{ Collagen III } & Forward & TTATAАACСААССТСТТССТ & 255 \\
\hline & Reverse & TATTATAGCACCATTGAGAC & \\
\hline \multirow[t]{2}{*}{ BIGLYCAN } & Forward & GATGGCCTGAAGCTCAA & 407 \\
\hline & Reverse & GGTTTGTTGAAGAGGCTG & \\
\hline \multirow[t]{2}{*}{ TENASCIN } & Forward & CGTGAAAAACAATACCCGAGGC & 160 \\
\hline & Reverse & GCCGTAGGAGAGTTCAATGCC & \\
\hline \multirow[t]{2}{*}{ GAPDH } & Forward & TCACCATCTTCCAGGAGCGA & 293 \\
\hline & Reverse & CACAATGCCGAAGTGGTCGT & \\
\hline
\end{tabular}




\subsection{Determination of the collagen content}

The amount of deposited collagen in the scaffold was quantified using a collagen assay kit, following the manufacturer's protocol (Jiancheng Inc., Nanjing, China). Briefly, lyophilized tissue specimens weighing about $3 \mathrm{mg}$ ( $\mathrm{n}=5$ for each group) were digested with a hydrolysis regent at $95{ }^{\circ} \mathrm{C}$ for $20 \mathrm{~min}$. Serial dilutions of hydroxyproline provided by the kit were utilized to construct a standard curve. A microplate reader was then used to measure the hydroxyproline concentration through absorbance readings at $550 \mathrm{~nm}$. The total collagen content was calculated from the hydroxyproline concentration using a conversion factor of 1:7.46 [15].

\subsection{Transmission electron microscopy (TEM)}

Tissue specimens at the 8 and 12 weeks time-points ( $n=3$ for each group) were processed following the standard procedures for TEM. Briefly, specimens were pre-fixed with $2 \%(\mathrm{w} / \mathrm{v})$ glutaraldehyde for $24 \mathrm{~h}$ at $4{ }^{\circ} \mathrm{C}$ and washed twice with PBS followed by post-fixation treatment with $1 \%(\mathrm{w} / \mathrm{v})$ osmic acid for $2 \mathrm{~h}$ at $4{ }^{\circ} \mathrm{C}$. After two washes in PBS, the specimens were dehydrated in an increasing ethanol gradient $(50-100 \% \mathrm{v} / \mathrm{v})$. The specimens were then filtrated in a 1:2 mixture of Epon-Araldite embedding resin and propylene oxide for $24 \mathrm{~h}$, followed by a $1: 1$ mixture of resin and propylene oxide for $24 \mathrm{~h}$, and a 2:1 mixture of resin and propylene oxide. Finally, the specimens were filtrated in $100 \%$ EponAraldite within molds for $48 \mathrm{~h}$ at $60{ }^{\circ} \mathrm{C}$. The embedded specimens were thin sectioned $(0.1 \mathrm{~mm})$ and viewed under TEM (Quanta 10 FEI). Approximately 500 collagen fibrils were imaged and measured for each sample to obtain an accurate representation of the fibril diameter distribution, using image analysis software (Image-Pro Plus, Rockville, MD, USA).

\subsection{Biomechanical evaluation}

Mechanical testing was performed using an Instron tension/compression system with Fast-Track software (Model 5543, Instron, Canton, MA) as described previously [13,25]. Single
A

\section{Unidirectional freezing}

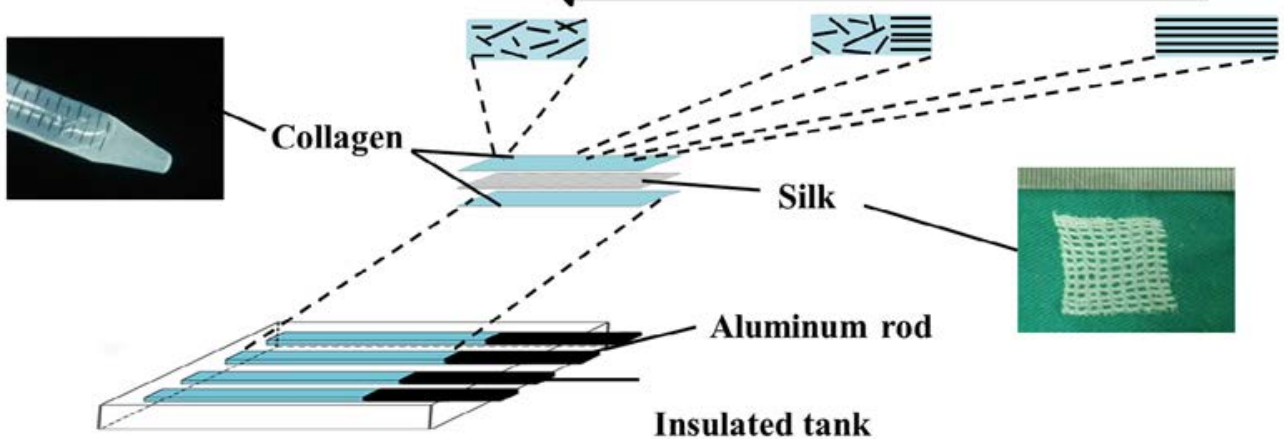

B
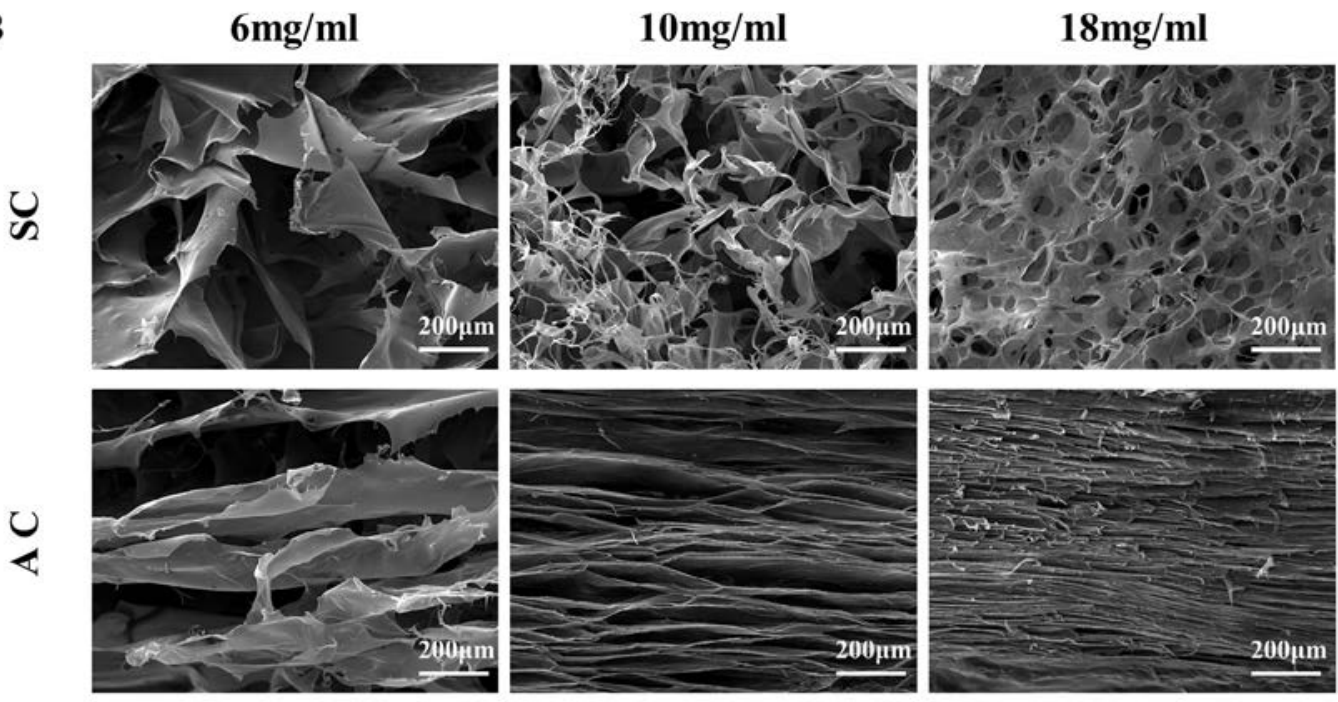

C

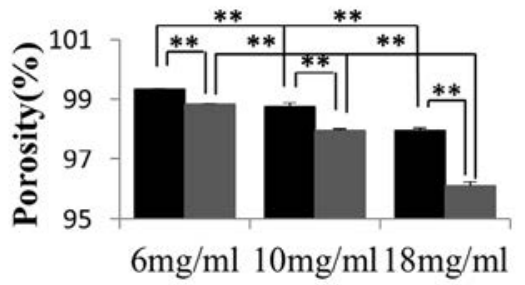

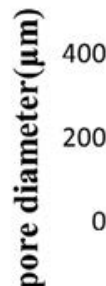

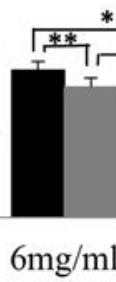

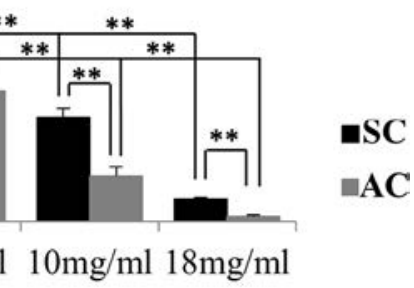

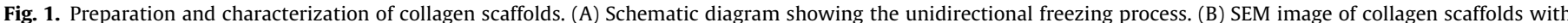

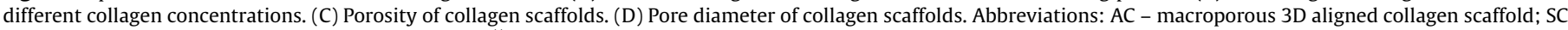
- sponge collagen scaffold. Statistically significant at ${ }^{* *} \mathrm{p}<0.01$. Scale bars: $200 \mu \mathrm{m}$ (B) 
pull-to-failure analysis was performed on collagen scaffolds and collagen/silk scaffolds ( $\mathrm{n}=5$ for each group). For rotator cuff tendon specimens at 12 weeks post-surgery ( $n=5$ for each group), the humerus head was secured by a specially designed restraining jig and the tendon end was pinched by vacuum with a clamp which had a rough surface (sandpaper), in order to reduce slippages. The tendon was positioned within the line of the applied tensile force. After applying a preload of $1 \mathrm{~N}$ with a test speed of $5 \mathrm{~mm} / \mathrm{min}$, the rotator cuff-humerus head complex underwent preconditioning for a total of 20 cycles. This was followed by load to failure testing at an elongation rate of $5 \mathrm{~mm} / \mathrm{min}$. The biomechanical parameters of the rotator cuff-humerus being measured included maximum force $(\mathrm{N})$, stress at failure (MPa), emerge $(\mathrm{mJ})$, stiffness $(\mathrm{N} / \mathrm{mm})$ and modulus (MPa).

\subsection{Statistical analysis}

All quantitative data were expressed as mean \pm SD. Student's $t$ test was performed to assess statistical significant differences of data between two groups. The threshold of significance was set at a value of $\mathrm{p}<0.05$.
A
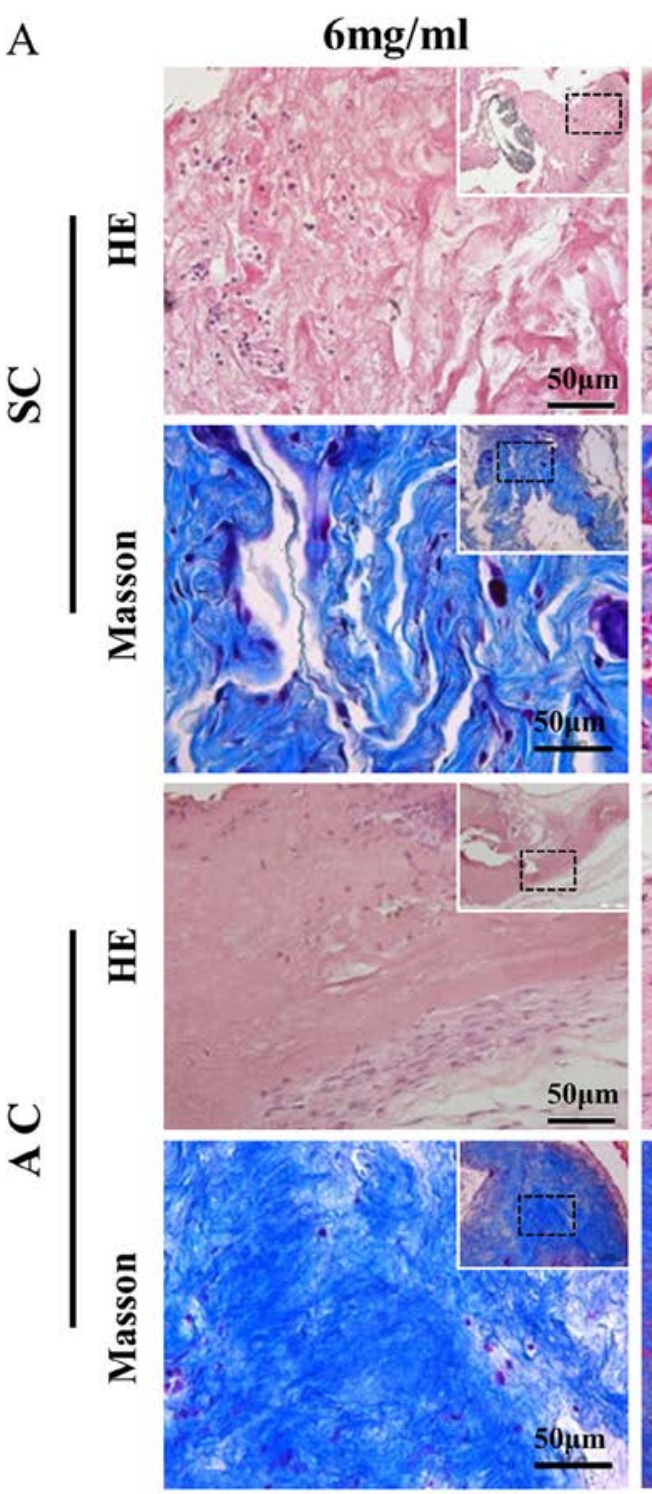

$10 \mathrm{mg} / \mathrm{ml}$
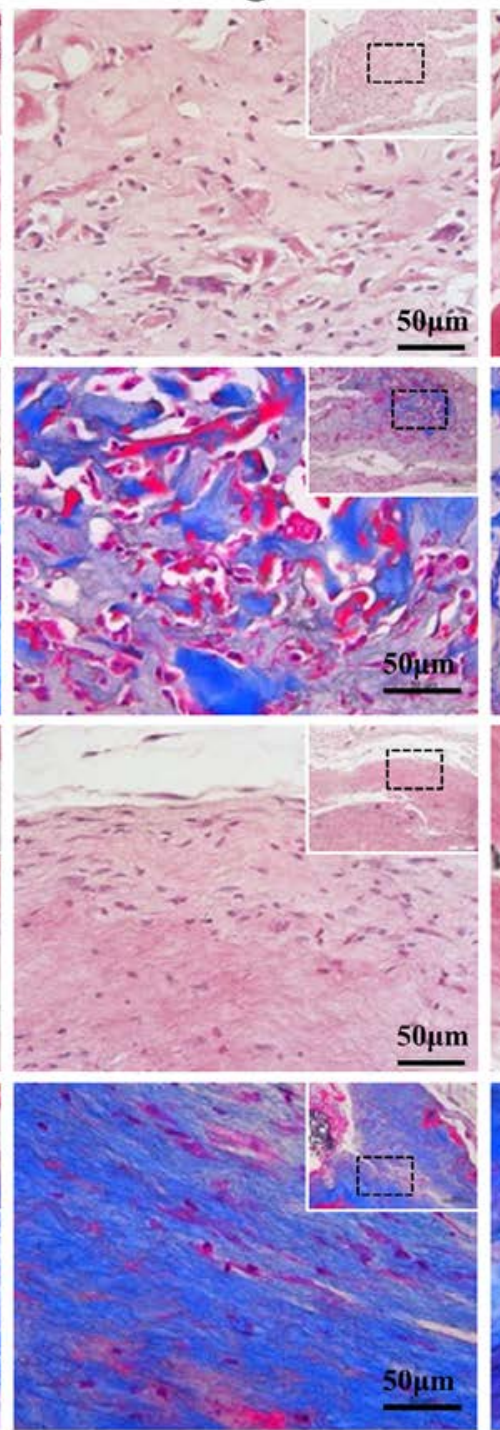

$18 \mathrm{mg} / \mathrm{ml}$
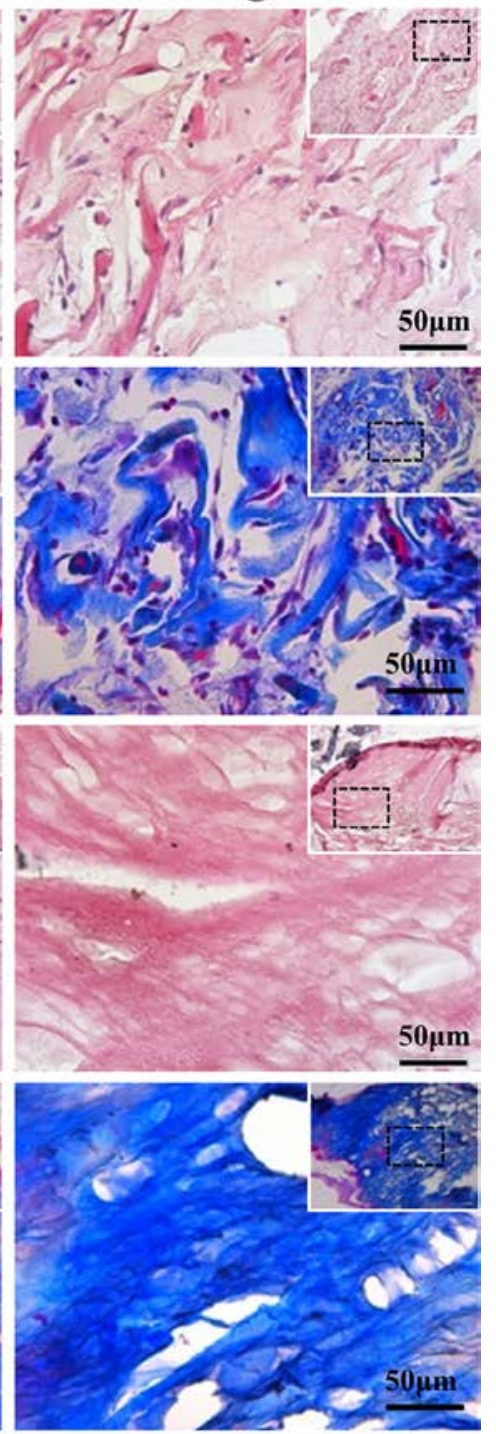

B

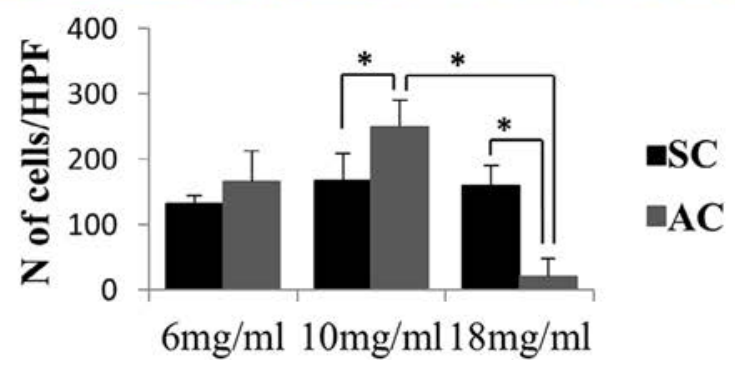

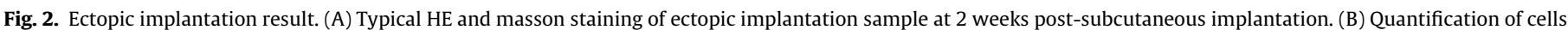

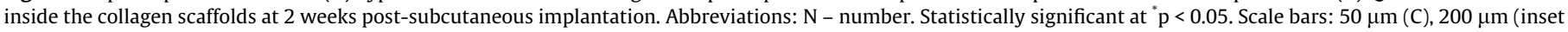
of C). 


\section{Results}

\subsection{Fabrication and characterization of collagen scaffolds}

Macroporous 3D aligned collagen scaffolds were fabricated successfully by unidirectional freezing technology (Fig. 1A). In order to optimize porosity and collagen concentration, three different concentrations $(6,10$, and $18 \mathrm{mg} / \mathrm{ml})$ were examined. SEM (upper surface) revealed that the spacing between collagen fibers became smaller with increasing concentrations of collagen, and that the macroporous 3D aligned collagen scaffold had a smaller pore diameter with the same collagen concentration compared with the sponge collagen/silk scaffold (310.29 \pm 15.07 vs. $\quad 350.66 \pm 21.87 \mu \mathrm{m}, \quad \mathrm{p}<0.01, \quad 6 \mathrm{mg} / \mathrm{ml} ; \quad 108.43 \pm 7.25$ vs. $247.61 \pm 21.68 \mu \mathrm{m}, \quad \mathrm{p}<0.01, \quad 10 \mathrm{mg} / \mathrm{ml} ; \quad 10.47 \pm 3.31 \quad$ vs. $53.86 \pm 3.28 \mu \mathrm{m}, \mathrm{p}<0.01,18 \mathrm{mg} / \mathrm{ml}$ ) (Fig. 1D). The porosity was also lower in the 3D aligned collagen scaffold (98.81 \pm 0.04 vs. $99.32 \pm 0.02 \%, p<0.01,6 \mathrm{mg} / \mathrm{ml} ; 97.94 \pm 0.08$ vs. $98.75 \pm 0.13 \%$, $\mathrm{p}<0.01, \quad 10 \mathrm{mg} / \mathrm{ml} ; \quad 96.08 \pm 0.14$ vs. $97.94 \pm 0.10 \%, \quad \mathrm{p}<0.01$, $18 \mathrm{mg} / \mathrm{ml}$ ) (Fig. 1C). Additionally, collagen fibers in the macroporous 3D aligned collagen scaffolds exhibited aligned distribution, unlike the disarranged random orientation observed in the colla- gen sponge scaffold (Fig. 1B). At 2 weeks post-subcutaneous implantation, more cellular infiltration could be observed in both the macroporous 3D aligned collagen scaffold and collagen sponge scaffold at $10 \mathrm{mg} / \mathrm{ml}$ collagen concentration, with the macroporous 3D aligned collagen scaffold having more cells. In contrast, there were almost no cells to be found inside the macroporous 3D aligned collagen scaffold at $18 \mathrm{mg} / \mathrm{ml}$ collagen. On the other hand, with $6 \mathrm{mg} / \mathrm{ml}$ collagen, the scaffold exhibited signs of collapse, and it is difficult for cells to infiltrate within the collapsed region. Additionally, cells in the macroporous 3D aligned collagen scaffold displayed more aligned orientation than the collagen sponge scaffold (Fig. 2). Hence, we determined $10 \mathrm{mg} / \mathrm{ml}$ to be the optimal concentration for further studies.

\subsection{Characterization of collagen/silk scaffolds}

Macroporous 3D aligned collagen/silk scaffold and sponge collagen/silk scaffold were successfully fabricated. The sponge collagen/ silk scaffold has sponge-like surfaces (Fig. 3A), while the macroporous 3D aligned collagen/silk scaffold displayed a smoother and brighter white appearance (Fig. 3B). Light microscopy revealed that the collagen fibers of the macroporous $3 \mathrm{D}$ aligned collagen/silk
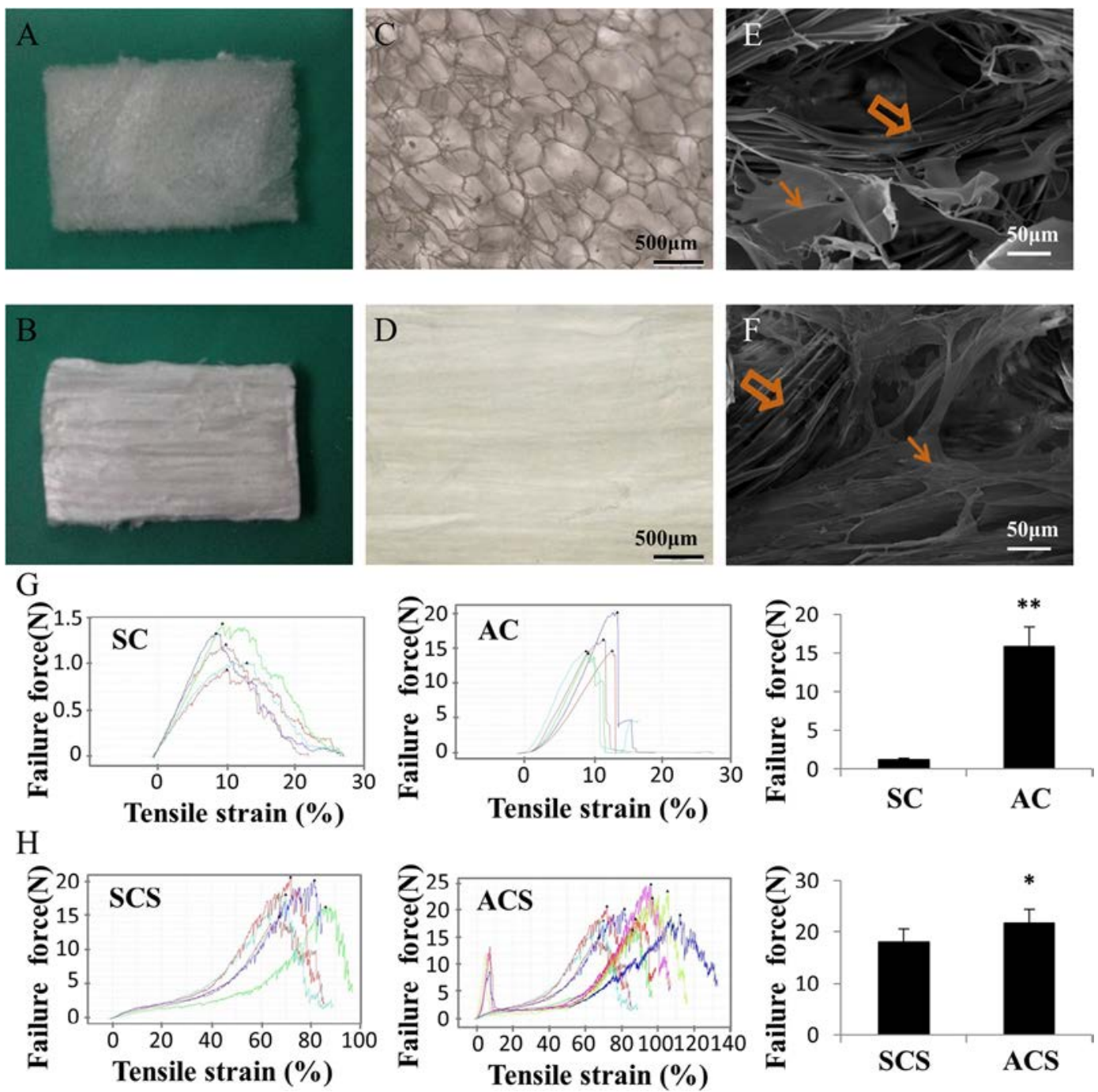

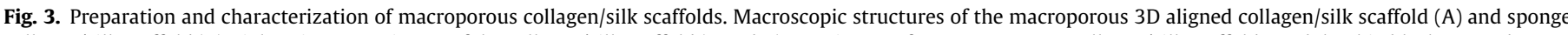

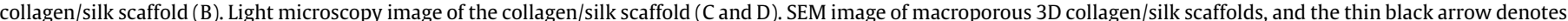

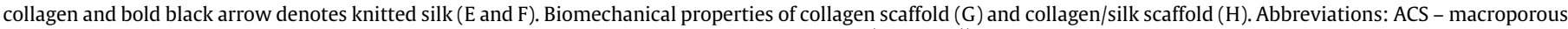
3D aligned collagen/silk scaffold; SCS - sponge collagen/silk scaffold. Statistically significant at ${ }^{*} \mathrm{p}<0.05,{ }^{* * *} \mathrm{p}<0.01$. Scale bars: $500 \mu \mathrm{m}$ (C and D), $50 \mu \mathrm{m}(\mathrm{E}$ and F). 
scaffold were mostly parallel to each other, while the sponge collagen/silk scaffold exhibited random and disordered orientations (Fig. 3C and D). SEM images demonstrated that the knitted silk was tightly integrated with collagen, with the bundles of silk and collagen fibers being alternately chained (Fig. 3E and F). (The failure force of the macroporous 3D aligned collagen scaffold was 13 -fold that of the collagen sponge scaffold $(15.90 \pm 2.45$ vs. $1.19 \pm 0.21 \mathrm{~N} ; \mathrm{p}<0.01$ ) (Fig. 3G). Additionally, the macroporous 3D aligned collagen/silk scaffold had a $19.7 \%$ increased failure force compared with the sponge collagen/silk scaffold (21.65 \pm 2.74 vs. $18.09 \pm 2.49 \mathrm{~N} ; \mathrm{p}<0.05$ ). Surprisingly, an obvious small peak appeared before the main peak in the failure force curve of the macroporous 3D aligned collagen/silk scaffold, which was attributed to breakage of collagen fibers (Fig. $3 \mathrm{H}$ ).

\subsection{Cell proliferation and arrangement on the collagen/silk scaffolds}

In order to characterize cell-scaffold interactions, rTSPCs were seeded on collagen/silk scaffolds. $24 \mathrm{~h}$ after seeding, rTSPCs became elongated, and were oriented nearly parallel to the collagen substrate on the macroporous 3D aligned collagen/silk scaffold. By contrast, rTSPCs on the sponge collagen/silk scaffold exhibited polygonal morphology with random orientation (Fig. 4A and B). Additionally, rTSPCs on the macroporous 3D aligned collagen/silk scaffold were longer than that on the sponge collagen/silk scaffold ( $36.37 \pm 1.07$ vs. $24.40 \pm 1.83 \mu \mathrm{m} ; \mathrm{p}<0.01$ )
(Fig. 4C). SEM revealed that rTSPCs attached and spread well on both scaffolds. On day 3, matrix-like deposition could be observed, and rTSPCs on the macroporous 3D aligned collagen/silk scaffold were aligned along the collagen substrate, whereas rTSPCs on the sponge collagen/silk scaffold were randomly oriented. Cell sheets formed on the macroporous 3D aligned collagen/silk scaffold on day 7, which was similar to natural tendon morphology (SFig. 1). Although the surface alignment of cells and matrix was attenuated after 7 days of culture, this could be because cell-cell contact became the predominant influence on the direction of cellular alignment [1]. CCK-8 assays on days 1, 3 and 7 after seeding showed that TSPCs proliferated well, and there were no statistically significant differences between the two groups (Fig. 4D).

\subsection{Effects on rotator cuff repair}

Scaffolds were implanted within rabbit rotator cuff tendon and harvested at 4,8 and 12 weeks post-surgery (Fig. 5A and B). The regenerative tendons of both groups exhibited a cord-like shape and were filled with fibrous tissues that were similar to normal rotator cuff in shape, luster, and color (S-Fig. 2).

We assessed the effects of the macroporous 3D collagen/silk scaffold on rotator cuff repair through histology in different regions. At the junction site, continuous tissue formed from the tendon section in both groups at 4 weeks post-surgery. However, the regenerative tissue in the macroporous 3D aligned collagen/

A
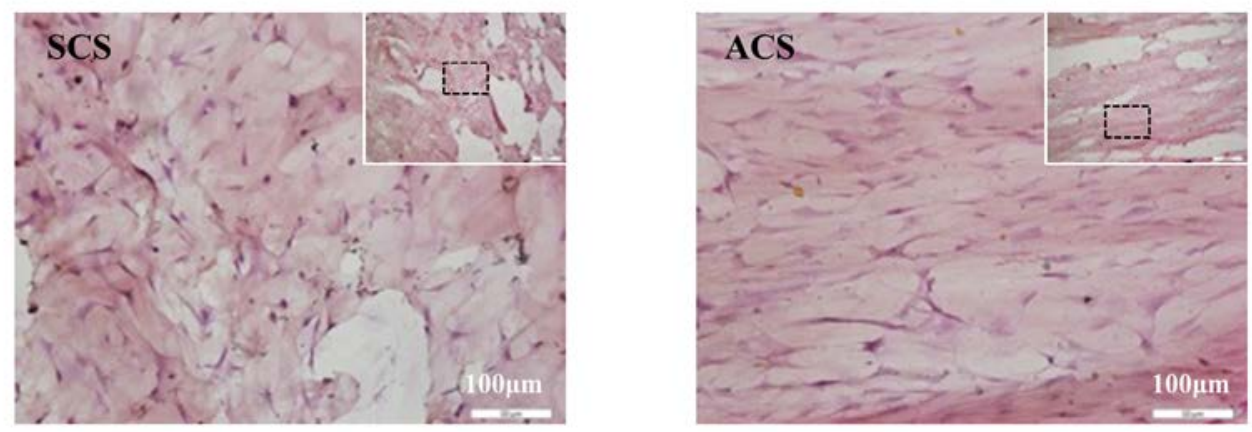

$\mathrm{B}$
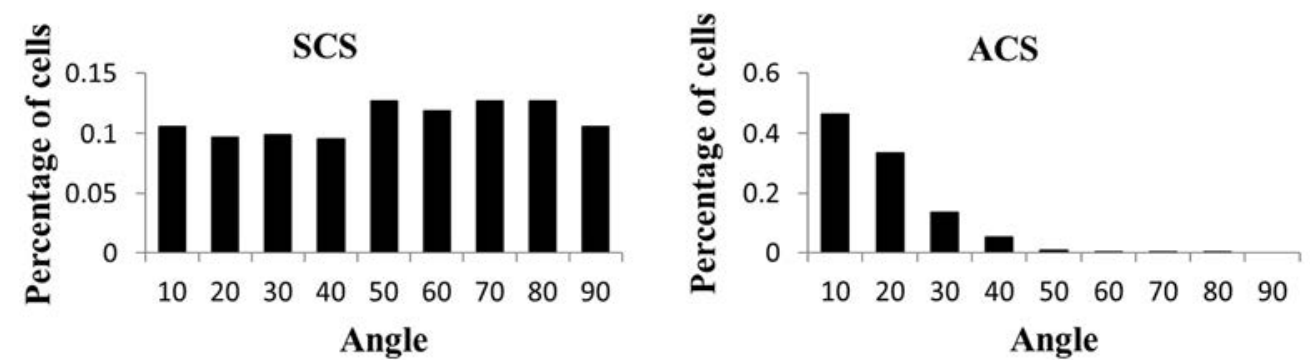

C

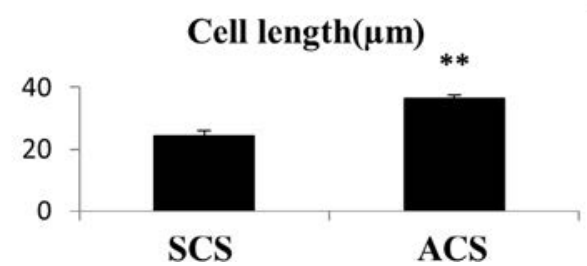

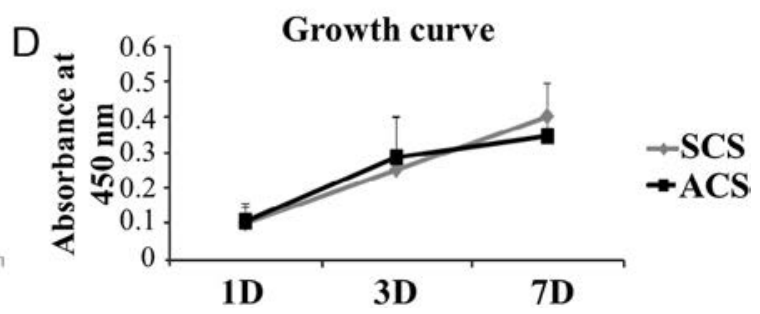

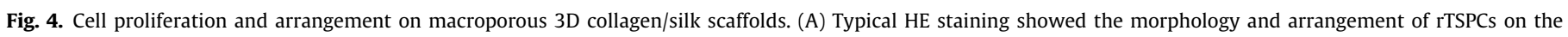

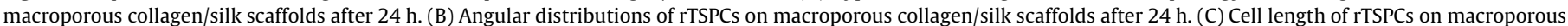

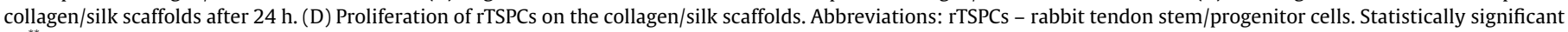
at ${ }^{* *} \mathrm{p}<0.01$. Scale bars: $100 \mu \mathrm{m}(\mathrm{A}), 500 \mu \mathrm{m}$ (inset of A). 

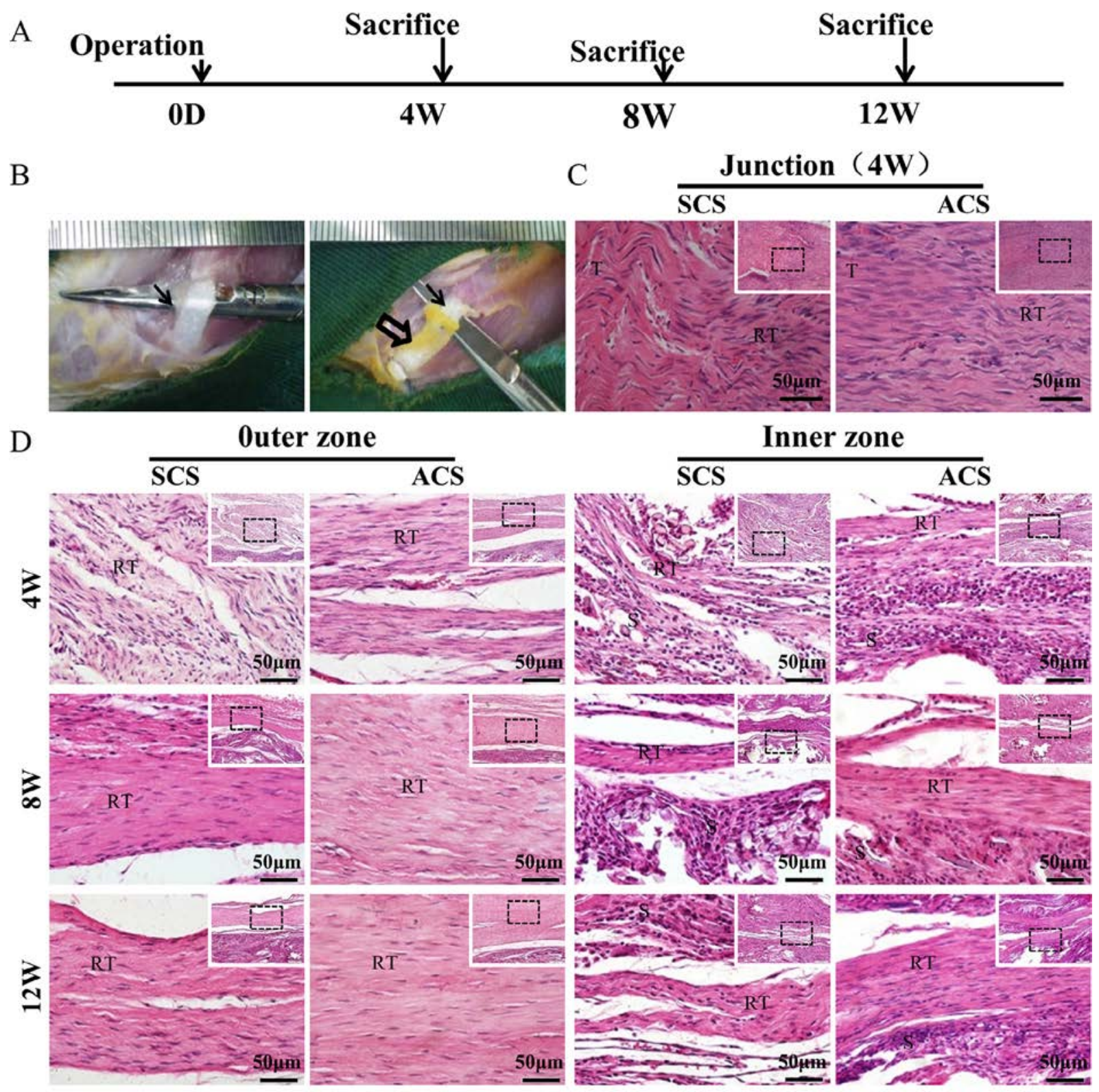

E

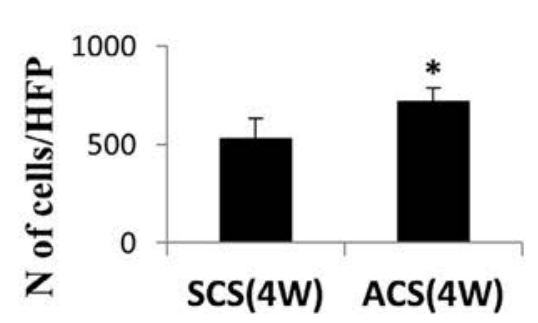

$\mathrm{F}$

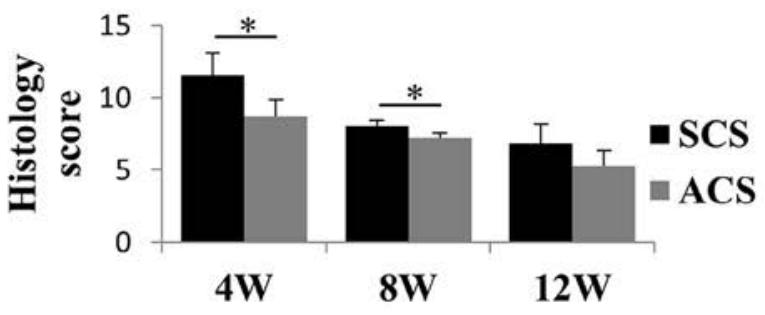

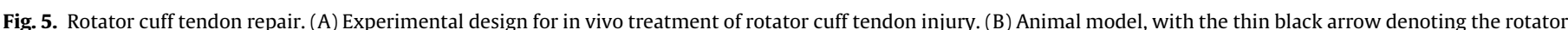

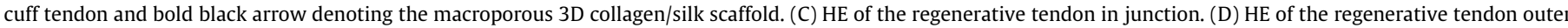

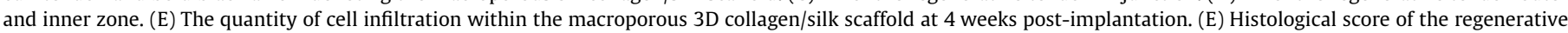

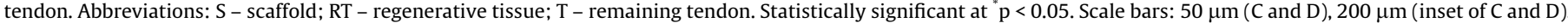

silk scaffold was more organized, and more ovoid cells were present (Fig. 5C). Additionally, there were abundant organized bundles of collagen fibers being formed in the outer zone of the macroporous 3D aligned collagen/silk scaffold. By contrast, the regenerative connective tissues were more disorganized and immature on the surface of the sponge collagen/silk scaffold. Inside the macroporous 3D aligned collagen/silk scaffold, dense regenerative connective tissues were formed, and organized bundles of collagen fibers could be observed. In contrast, there were only sporadic tiny collagen fibers inside the sponge collagen/silk scaffold (Fig. 5D). Additionally, more cells could be observed inside the macroporous 3D aligned collagen/silk scaffold compared to the sponge collagen/silk scaffold ( $714.56 \pm 72.30$ vs. $530.75 \pm 99.56$ / HPF; $\mathrm{p}<0.05$ ) (Fig. 5E), which thus demonstrated that the macroporous 3D aligned topology could facilitate cell infiltration [26]. At 8 weeks post-surgery, the regenerative tissue became more 
mature in both groups, which could be verified by the presence of more spindle-shaped cells and less rounded cells, with the sponge collagen/silk scaffold group still lagging behind the macroporous 3D aligned collagen/silk scaffold group. At 12 weeks postimplantation, bundles of mature collagen fibers were observed on the surface of the macroporous 3D aligned collagen/silk scaffold, which more closely resembled natural tendon fibers (SFig. 3). Additionally, within the scaffold, more collagen fibers were formed upon degradation of the scaffold, and the regenerative tissue of the macroporous 3D aligned collagen/silk scaffold group were denser and more matured (Figs. 5D and 6A). It was interesting to observe that regeneration within the scaffold interior lagged behind that in the outer zone. This might be because there were more cells on the surface derived from surrounding tissues than that from the tendon resident tissue, and it took time for cells to infiltrate the scaffold [1].

Histology scoring showed that the macroporous 3D aligned collagen/silk group more closely resembled natural tendon $(8.73 \pm 1.10$ vs. $11.57 \pm 1.52, \mathrm{p}<0.05$, at 4 weeks post-surgery; $7.17 \pm 0.39$ vs. $8.03 \pm 0.39, \mathrm{p}<0.05$, at 8 weeks post-surgery; $5.24 \pm 1.08$ vs. $6.80 \pm 1.34$ at 12 weeks post-surgery) (Fig. $5 \mathrm{~F}$ ).

Polarized light microscopy was utilized to estimate the maturity and organization of the regenerative collagen fibers. The results showed that collagen fibers in the macroporous $3 \mathrm{D}$ aligned
A

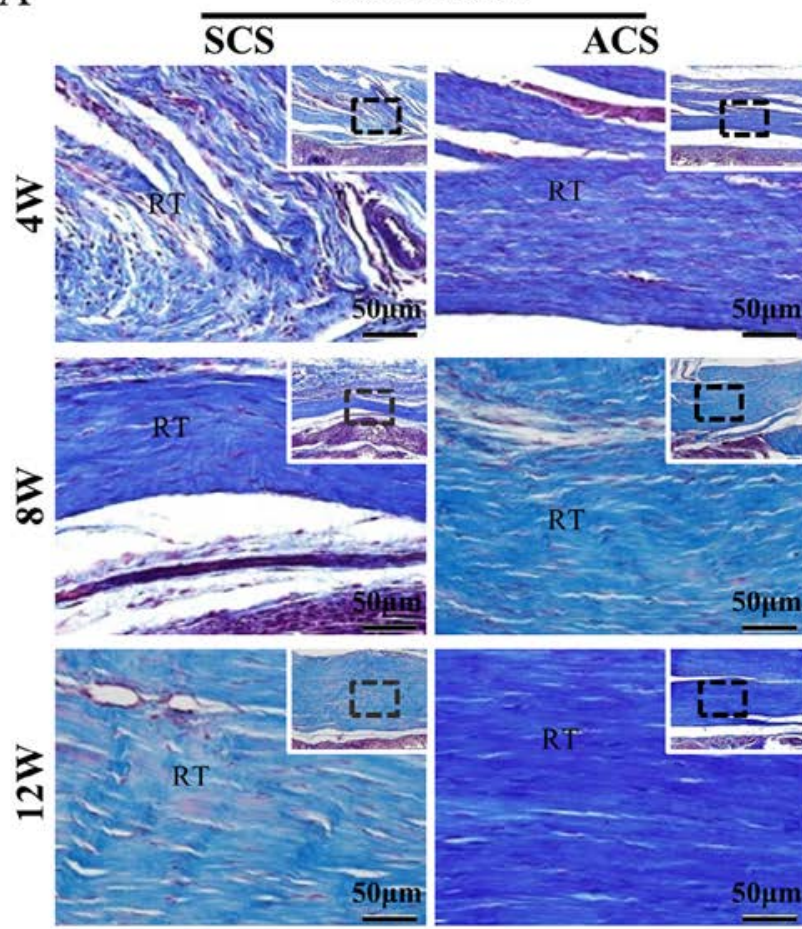

B

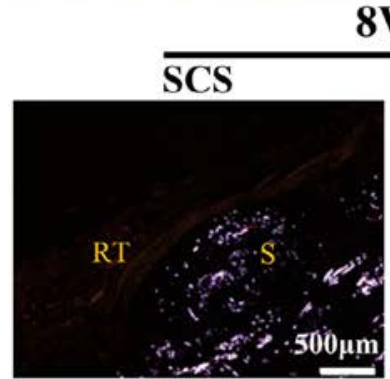

8W

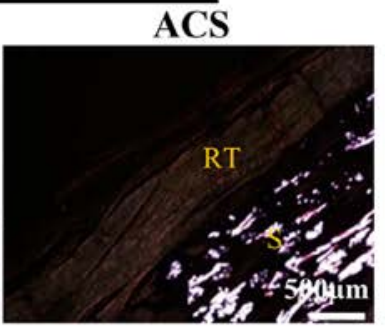

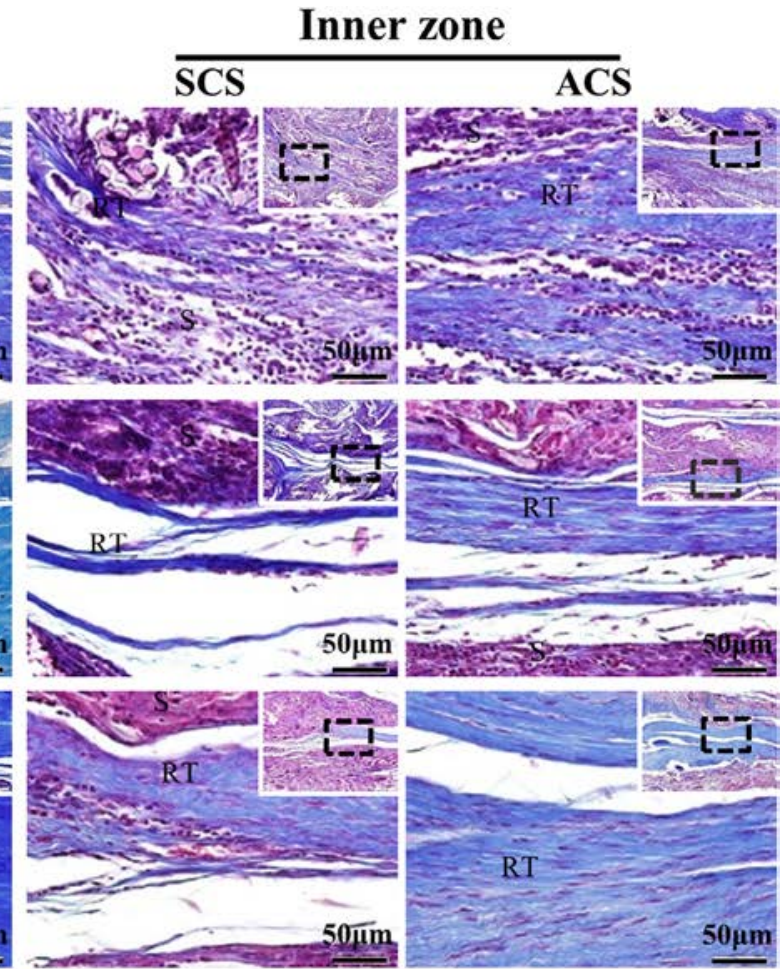

$12 \mathrm{~W}$

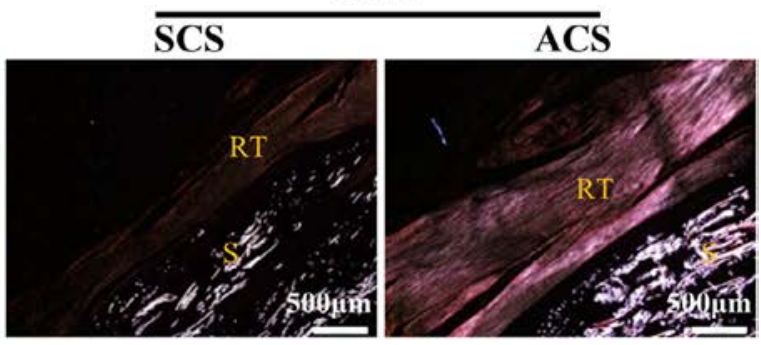

$\mathrm{C}$

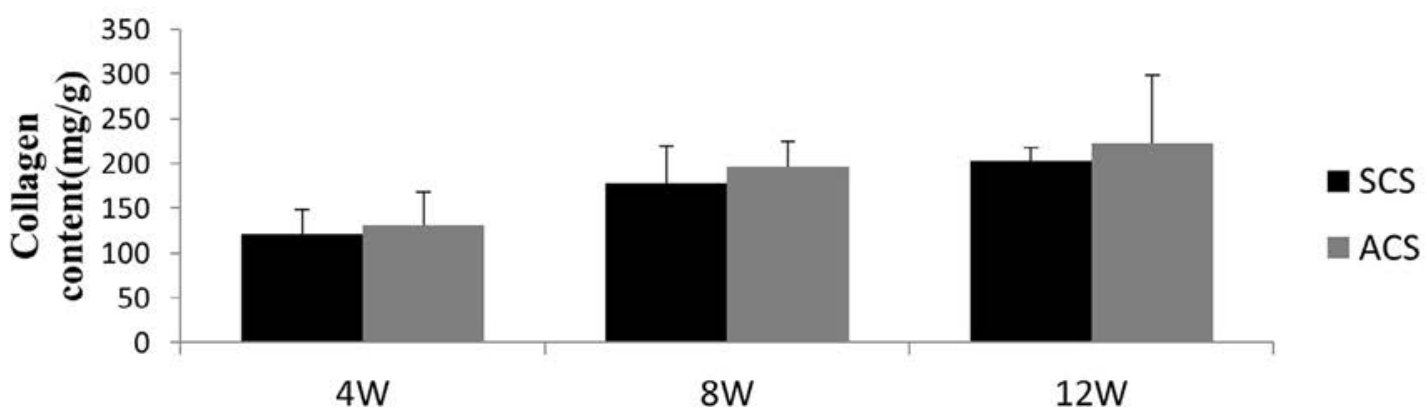

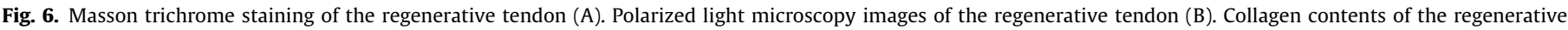
tendon (C). Scale bars: $50 \mu \mathrm{m}$ (A), $200 \mu \mathrm{m}$ (inset of A), $500 \mu \mathrm{m}$ (B). 
collagen/silk scaffold were obviously detectable by 8 weeks postsurgery. At 12 weeks post-surgery, lighter and more abundant collagen fibers could be observed in the macroporous 3D aligned collagen/silk scaffold, which closely resembled natural tendon fibers (S-Fig. 3). By contrast, in the sponge collagen/silk scaffold, collagen fibers were sparsely present at 8 weeks post-surgery, and still lagged behind the macroporous 3D aligned collagen/silk scaffold group at 12 weeks post-surgery (Fig. 6B).

\subsection{Gene expression in repaired rotator cuff tendon}

Tendon repair related genes, including collagen I (col I), collagen III (col III), tenascin (TCN) and biglycan (BGN), were analyzed by quantitative PCR. At 4 weeks post-surgery, the data showed that the transcript levels of col I, col III, TCN and BGN of the macroporous 3D aligned collagen/silk scaffold group were higher than the sponge collagen/silk scaffold group by $1.68,1.70(\mathrm{p}<0.05)$, 1.79 and $2.92(\mathrm{p}<0.05)$-folds respectively (Fig. 7A). However, these differences diminished by 8 weeks post-surgery because of the degradation of the collagen scaffold, and there were no statistically significant differences between the two groups (Fig. 7B). Furthermore, at 12 weeks post-surgery, the expression of BGN in the macroporous 3D aligned collagen/silk scaffold group was only $0.40(p<0.05)$ fold of that in the sponge collagen/silk scaffold group, and expression levels of col I, col III, and DCN were not significantly different between the two groups (Fig. 7C).

\subsection{Formation of collagen fibrils}

The formation of collagen fibrils were assessed as evidence of the reconstruction and maturation of the regenerative rotator cuff tendon. At any time point, the macroporous 3D aligned collagen/ silk group displayed consistently higher collagen content than the sponge collagen/silk group (131.12 \pm 36.73 vs. $121.33 \pm$ $27.13 \mathrm{mg} / \mathrm{g}$ at 4 weeks; $196.24 \pm 27.68$ vs. $177.39 \pm 41.46 \mathrm{mg} / \mathrm{g}$ at 8 weeks post-surgery; $222.48 \pm 75.44$ vs. $202.71 \pm 14.71 \mathrm{mg} / \mathrm{g}$ at
12 weeks post-surgery) (Fig. 6C). However, these observed differences were not statistically significant because of the presence of silk in the regenerative tendon and the small sampling size.

Ultrastructural morphology of regenerative collagen fibers were analyzed by TEM imaging. It was demonstrated that larger and denser collagen fibers were formed in the macroporous 3D aligned collagen/silk scaffold, while the collagen fibers in the sponge collagen/silk scaffolds were thin and sparse (Fig. 8A). The distribution of regenerative collagen fibers in the macroporous 3D aligned collagen/silk scaffold group was more uniform than the sponge collagen/silk scaffold group. Additionally, the macroporous 3D aligned collagen/silk scaffold group had larger diameter collagen fibrils than the sponge collagen/silk group at 8 and 12 weeks postsurgery $(48.77 \pm 3.31$ vs. $33.84 \pm 2.99 \mathrm{~nm}, \mathrm{p}<0.05$, at 8 weeks; $48.72 \pm 3.75$ vs. $44.26 \pm 5.03 \mathrm{~nm}$ at 12 weeks) (Fig. 8 B).

\subsection{Biomechanical properties of repaired rotator cuff tendon}

Biomechanical properties were evaluated by the Instron tension/compression system. The macroporous 3D aligned collagen/silk scaffold group exhibited superior mechanical properties to the sponge collagen/silk scaffold group (Fig. 8C). The failure force of both groups were higher than native tendon (S-Fig. 3), and the macroporous 3D aligned collagen/silk groups was $41.13 \%$ higher than that of the sponge collagen/silk scaffold group $(139.85 \pm 49.36$ vs. $99.09 \pm 33.98 \mathrm{~N}, \mathrm{p}<0.05)$. The stress at failure of the macroporous 3D aligned collagen/silk groups was $26.53 \%$ higher than that of the sponge collagen/silk scaffold group ( $4.34 \pm 1.56$ vs. $3.43 \pm 1.88 \mathrm{MPa}, \mathrm{p}=0.05$ ) (S-Fig. 4 ). The energy of the macroporous 3D aligned collagen/silk groups was $61.54 \%$ higher than that of the sponge collagen/silk scaffold group $(0.42 \pm 0.15$ vs. $0.26 \pm 0.11 \mathrm{~J}, \mathrm{p}<0.05)$. The stiffness and modulus were not significantly different between the two groups because of the presence of silk in the regenerative tendon $(25.50 \pm 10.36$ vs. $\quad 34.35 \pm 12.23 \mathrm{~N} / \mathrm{mm} ; \quad 26.77 \pm 12.12$ vs. $24.56 \pm 12.72$ ) (S-Fig. 4).
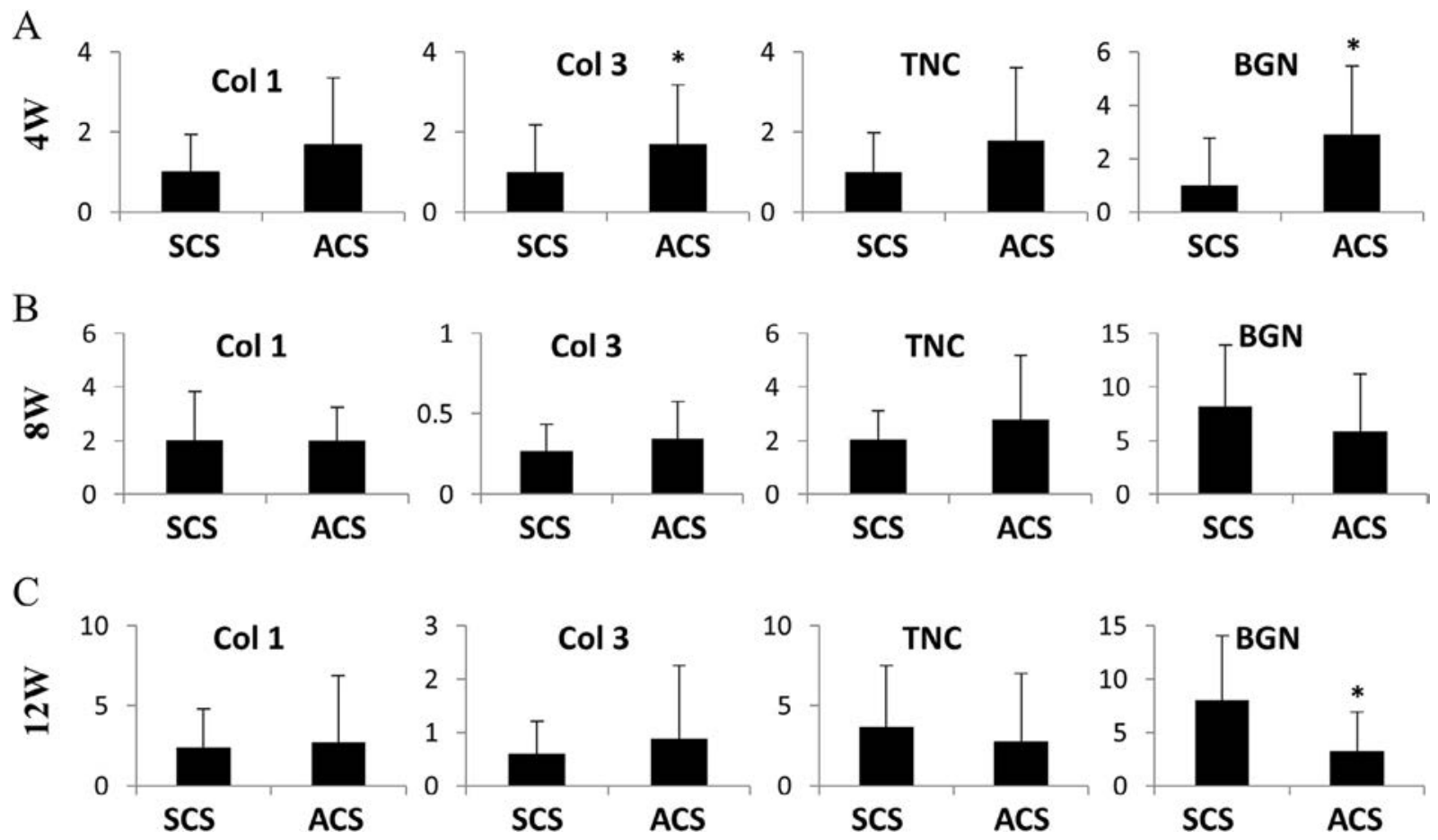

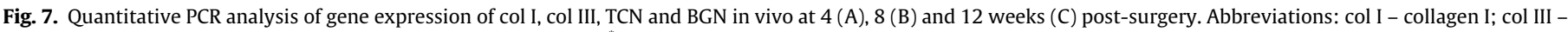
col III; TCN - tenascin; BGN - biglycan. Statistically significant at ${ }^{*} \mathrm{p}<0.05$. 


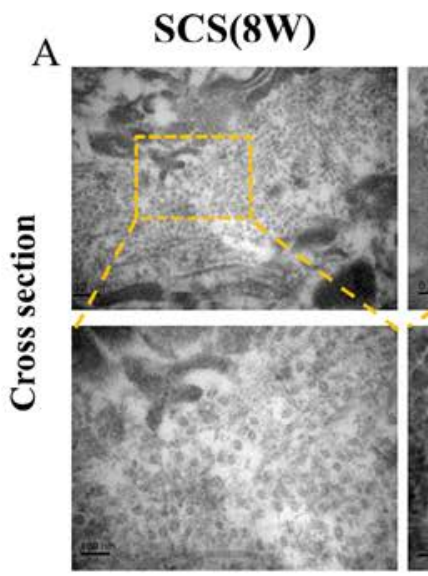

$\operatorname{ACS}(8 W)$
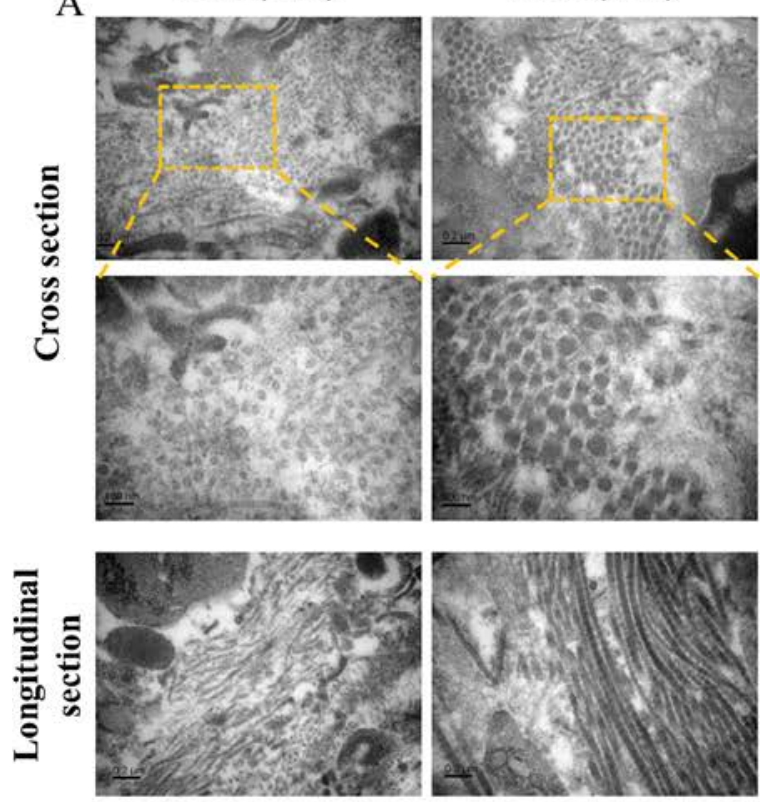

B

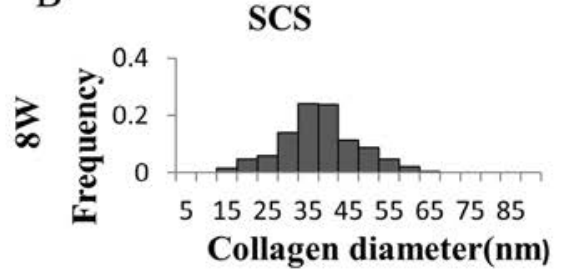

SCS

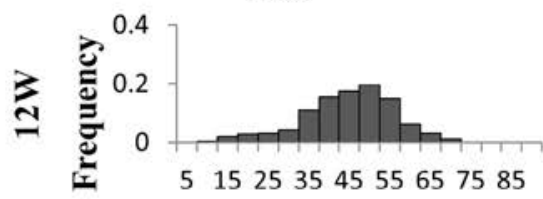

$\mathrm{C}$

Collagen diameter(nm)
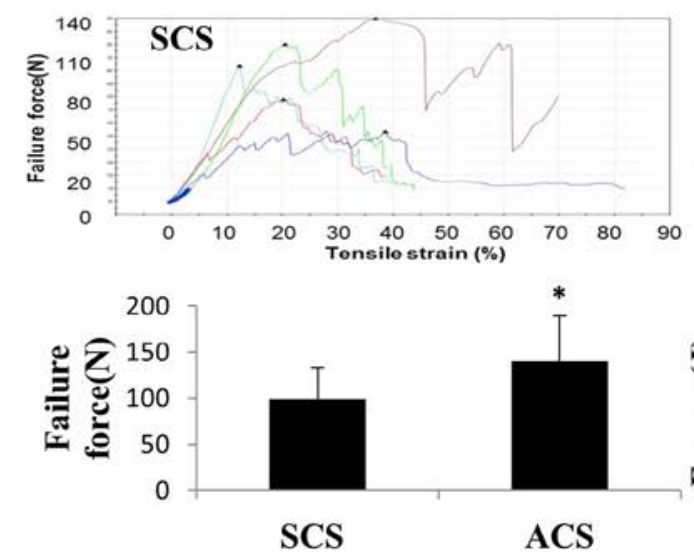

$\operatorname{SCS}(12 W)$
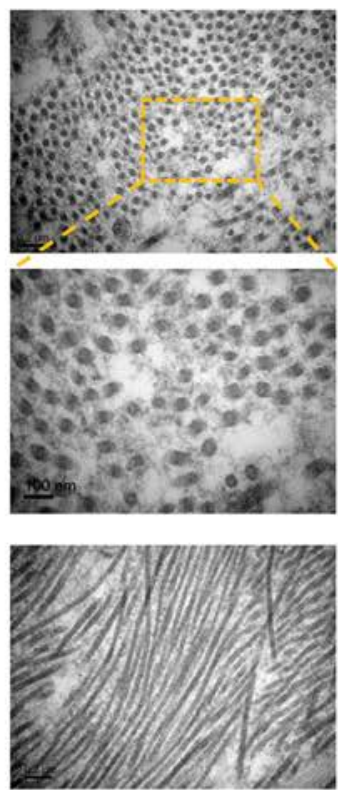

ACS
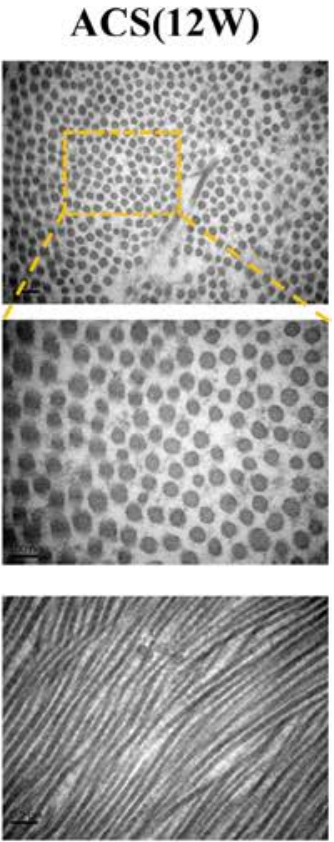

Collagen diameter(nm)

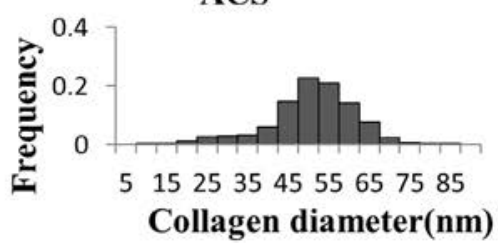

ACS

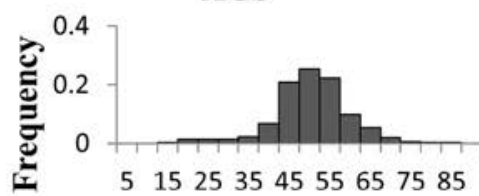

Collagen diameter(nm)

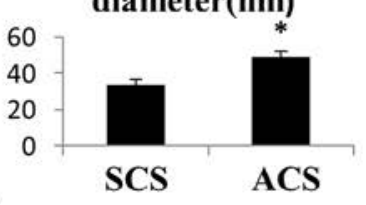

Collagen diameter(nm)
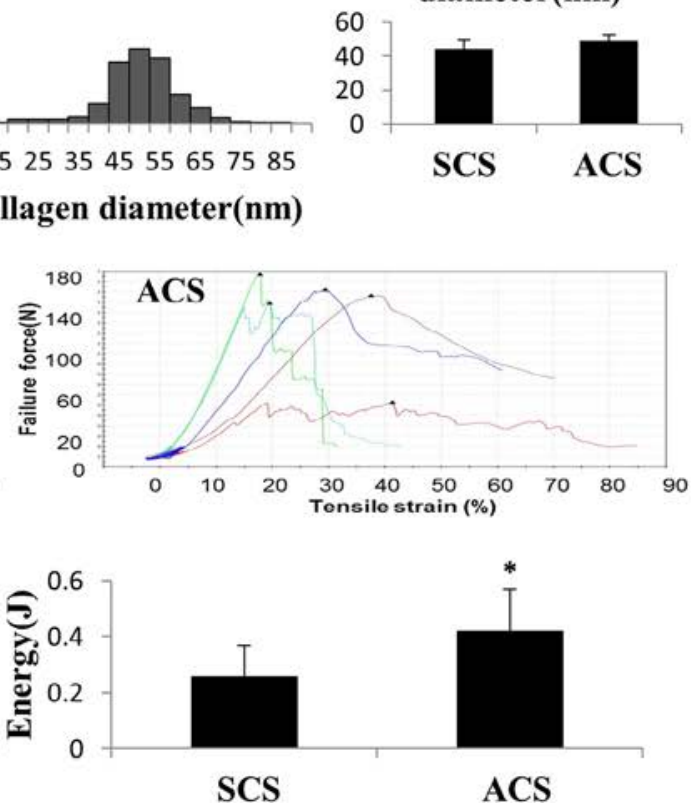

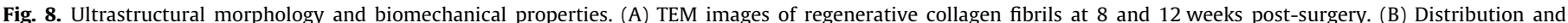

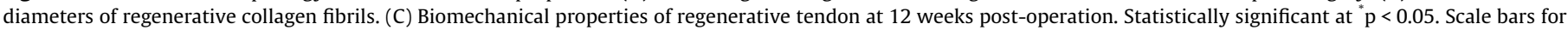
the first three rows: $0.2 \mu \mathrm{m}, 100 \mathrm{~nm}$ and $0.2 \mu \mathrm{m}$.

\section{Discussion}

In this study, we fabricated a novel macroporous 3D aligned collagen/silk scaffold based on our previous studies, combining aligned collagen sponge with knitted silk, which significantly enhanced massive rotator cuff repair. The macroporous 3D aligned bionic collagen/silk scaffold was successfully fabricated by unidirectional freezing technology, integrating collagen and knitted silk tightly. Subsequently, we found that $10 \mathrm{mg} / \mathrm{ml}$ was the optimal collagen concentration that yielded the appropriate porosity 
conducive for cell infiltration from the tissue environment upon ectopic implantation. We also demonstrated that the macroporous 3D aligned topology had a profound influence on the cellular morphology, arrangement and self-organization of rTPSCs, which resulted in the cell-scaffold complex exhibiting an aligned supracellular structure similar to natural tendon in morphology. We found that the regenerative $\mathrm{RC}$ tendon with the macroporous $3 \mathrm{D}$ aligned bionic collagen/silk scaffold had larger diameter collagen fibrils, deposited more organized extracellular matrix, and exhibited better mechanical properties. Collectively, these results indicated that the macroporous 3D aligned collagen/silk scaffold can have practical clinical applications for tendon tissue engineering in the near future.

Collagen is a major component of the extracellular matrix, and has frequently been utilized as a scaffold biomaterial [11]. In previous studies, sponge collagen/silk scaffold with seeded cells were utilized for repairing tendon/ligament injuries, with obvious positive efficacy $[13,22,25,27]$. In this study, the sponge collagen/silk scaffold again yielded consistent positive efficacy in rotator cuff tendon repair, by regulating cell adhesion, spreading and differentiation. However, the structure and function of regenerative tendons in the sponge collagen/silk scaffold were still much inferior to that of natural tendons because of the randomly aligned fibers of sponge collagen. To overcome this limitation, we fabricated the macroporous 3D aligned collagen/silk scaffolds by unidirectional freezing technology. SEM indicated that the collagen and knitted silk were integrated tightly. Our study demonstrated the influence of the macroporous 3D aligned scaffold on the arrangement of rTSPC and tendon repair. The in vitro results demonstrated that rTSPCs cultured on macroporous 3D aligned collagen/silk scaffolds became elongated like mature tendon cells, and was aligned with the collagen fibers as early as day 1 . After 7 days of culture, the cells-scaffold complex was similar to native tendon in morphology. In vivo, the macroporous 3D aligned collagen scaffold induced the regenerative tissue to be aligned like natural tendon. By contrast, the regenerative tissue was disordered in the randomly aligned sponge collagen/silk scaffold group, even though there was abundant collagen deposition at 4 weeks post-surgery.

A previous study reported that collagen concentration could influence the pore size and porosity, and that higher concentrations resulted in smaller pore sizes and lower porosity [24]. In our study, we observed the same results, both for the aligned collagen/silk scaffold and randomly aligned sponge collagen/silk scaffold, with the pore size and the porosity decreasing with increasing collagen concentration. We fabricated collagen scaffolds with different concentrations resulting in different pore sizes and porosities and tested these by ectopic implantation with pressure from surrounding tissues. It was demonstrated that low concentration scaffolds could not sustain their structural integrity and easily collapsed, and that the cells could not infiltrate the collapsed region, similar to that reported by another study [7]. On the other hand, the high concentration scaffold was too compact for cells to infiltrate, because pore sizes of several hundred micrometers and porosity of $90 \%$ or higher are conducive for cell infiltration [20]. Additionally, porosity is useless without connectivity between the surface pores and interior of the scaffold. Collectively, macroporous 3D collagen scaffolds fabricated by unidirectional freezing technology with a concentration of $10 \mathrm{mg} / \mathrm{ml}$ collagen was found to have optimal macroporous pore size $(108.43 \pm 7.25 \mu \mathrm{m})$, porosity $(97.94 \pm 0.075 \%)$ and mechanical stability $(21.65 \pm 2.74 \mathrm{~N})$ for rabbit rotator cuff tendon repair. Continuous tissue formation from the tendon section could be observed at the junction site in both groups. Furthermore, the ectopic study, together with the results at 4 weeks post-surgery in situ, both proved that there were more cells in the macroporous 3D aligned collagen/silk scaffold than the sponge collagen/silk scaffold. This suggested that the macroporous
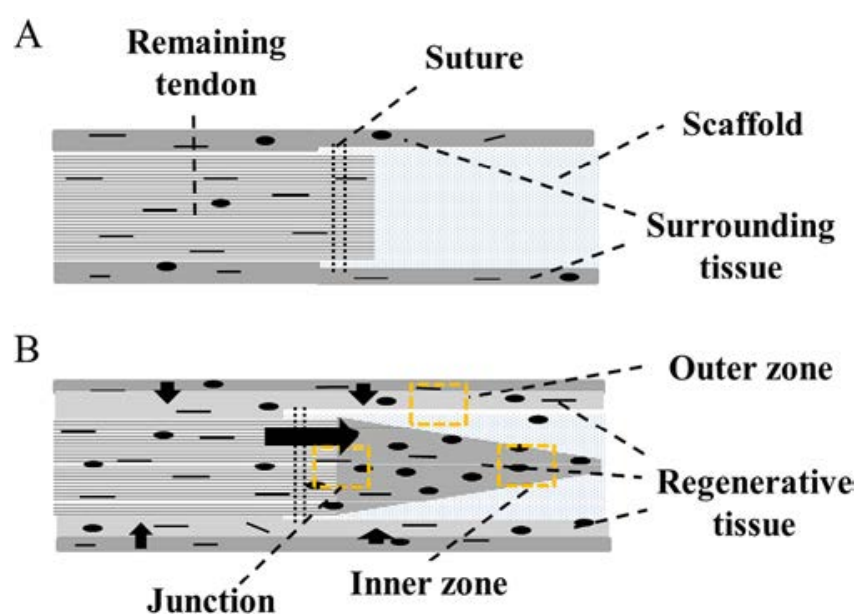

Fig. 9. Schematic diagram of the animal model (A) and the regenerative tissue (B).

3D aligned collagen/silk scaffold can recruit cells more effectively from both the tendon itself and the surrounding tissues (Fig. 9).

There are still some limitations to this study. One such limitation is that the collagen scaffold degraded too quickly in vivo within the rabbit model, and almost no intact collagen scaffold could be found even after 4 weeks post-surgery. Advanced fabrication technology such as crosslinking with gold nanoparticles [28], could be utilized to slow down the collagen degradation rate. However, the biodegradation rate of the collagen matrix should also match the deposition of new tissues in situ. Additionally, the deposition rate will depend on the inflammatory reaction, blood supply to the tissue and the animal species. These thus imply that the optimal collagen biodegradation rate in the rabbit model may not be optimal for humans. Hence, the same results need to be demonstrated in a larger animal tendinopathy model (such as pigs and monkeys) before human clinical application.

\section{Conclusions}

In summary, our study developed a macroporous 3D aligned collagen/silk scaffold for massive rotator cuff repair by inducing aligned supracellular structures similar to natural tendon, which in turn enhanced cellular infiltration and tenogenic differentiation. The positive efficacy of the macroporous 3D aligned collagen fibers in providing a conducive healing environment for endogenous cell migration and differentiation of stem/progenitor cells from both the tendon itself and surrounding tissues was conclusively demonstrated, which suggests much potential for clinical applications.

\section{Acknowledgement}

This work was supported by the National High Technology Research and Development Program of China (863 Program) (2012AA020503), International Science \& Technology Cooperation Program of China (2015DFG32130), the National Natural Science Foundation of China $(81330041,81125014,31271041,81401781$, 81201396, 81271970, J1103603, 81572115, 81572157, 81171703, 31570987, 81522029), Zhejiang Provincial Natural Science Foundation of China (LR14H060001), Zhejiang Province Grants (LY14H060003, LY12H06006), Medical Science and Technology Project of Zhejiang Province (201341741), Regenerative Medicine in Innovative Medical Subjects of Zhejiang Province, Medical and Health Science and Technology Plan of the Department of Health of Zhejiang Province (2013RCA010), the Postdoctoral Foundation of China (2014M561775, 2014M551759), SSSTC program (EG 04- 
032014), and the Key Scientific and Technological Innovation Team of Zhejiang Province (2013TD11).

\section{Appendix A. Supplementary data}

Supplementary data associated with this article can be found, in the online version, at http://dx.doi.org/10.1016/j.actbio.2017.01. 041.

\section{References}

[1] S.B. Orr, A. Chainani, K.J. Hippensteel, A. Kishan, C. Gilchrist, N.W. Garrigues, D. S. Ruch, F. Guilak, D. Little, Aligned multilayered electrospun scaffolds fo rotator cuff tendon tissue engineering, Acta Biomater. 24 (2015) 117-126.

[2] M.D. McElvany, E. McGoldrick, A.O. Gee, M.B. Neradilek, F.R. Matsen, Rotator cuff repair: published evidence on factors associated with repair integrity and clinical outcome, Am. J. Sports Med. 43 (2015) 491-500.

[3] J.A. Greenspoon, M. Petri, R.J. Warth, P.J. Millett, Massive rotator cuff tears: pathomechanics, current treatment options, and clinical outcomes, J. Shoulder Elbow Surg. 24 (2015) 1493-1505.

[4] J. Pan, G.M. Liu, L.J. Ning, Y. Zhang, J.C. Luo, F.G. Huang, T.W. Qin, Rotator cuff repair using a decellularized tendon slices graft: an in vivo study in a rabbit model, Knee Surg. Sports Traumatol. Arthrosc. 23 (2015) 1524-1535.

[5] E.T. Ricchetti, A. Aurora, J.P. Iannotti, K.A. Derwin, Scaffold devices for rotator cuff repair, J. Shoulder Elbow Surg. 21 (2012) 251-265.

[6] Z. Yin, X. Chen, T. Zhu, J.J. Hu, H.X. Song, W.L. Shen, L.Y. Jiang, B.C. Heng, J.F. Ji, H W. Ouyang, The effect of decellularized matrices on human tendon stem/ progenitor cell differentiation and tendon repair, Acta Biomater. 9 (2013) 9317-9329.

[7] D.P. Beason, B.K. Connizzo, L.M. Dourte, R.L. Mauck, L.J. Soslowsky, D.R. Steinberg, J. Bernstein, Fiber-aligned polymer scaffolds for rotator cuff repair in a rat model, J. Shoulder Elbow Surg. 21 (2012) 245-250.

[8] M. Younesi, A. Islam, V. Kishore, J.M. Anderson, O. Akkus, Tenogenic induction of human MSCs by anisotropically aligned collagen biotextiles, Adv. Funct. Mater. 24 (2014) 5762-5770.

[9] A.J. Lomas, C.N. Ryan, A. Sorushanova, N. Shologu, A.I. Sideri, V. Tsioli, G.C. Fthenakis, A. Tzora, I. Skoufos, L.R. Quinlan, G. O’Laighin, A.M. Mullen, J.L. Kelly, S. Kearns, M. Biggs, A. Pandit, D.I. Zeugolis, The past, present and future in scaffold-based tendon treatments, Adv. Drug Deliv. Rev. 84 (2015) 257-277.

[10] D. Gaspar, K. Spanoudes, C. Holladay, A. Pandit, D. Zeugolis, Progress in cellbased therapies for tendon repair, Adv. Drug Deliv. Rev. 84 (2015) 240-256.

[11] J. Glowacki, S. Mizuno, Collagen scaffolds for tissue engineering, Biopolymers 89 (2008) 338-344.

[12] S.A. Muller, L. Durselen, P. Heisterbach, C. Evans, M. Majewski, Effect of a simple collagen type I sponge for achilles tendon repair in a rat model, Am. J. Sports Med. 44 (2016) 1998-2004.
[13] X. Chen, Y.Y. Qi, L.L. Wang, Z. Yin, G.L. Yin, X.H. Zou, H.W. Ouyang, Ligament regeneration using a knitted silk scaffold combined with collagen matrix, Biomaterials 29 (2008) 3683-3692.

[14] Z. Yin, X. Chen, J.L. Chen, W.L. Shen, N.T. Hieu, L. Gao, H.W. Ouyang, The regulation of tendon stem cell differentiation by the alignment of nanofibers, Biomaterials 31 (2010) 2163-2175.

[15] Z. Yin, X. Chen, H.X. Song, J.J. Hu, Q.M. Tang, T. Zhu, W.L. Shen, J.L. Chen, H. Liu, B.C. Heng, H.W. Ouyang, Electrospun scaffolds for multiple tissues regeneration in vivo through topography dependent induction of lineage specific differentiation, Biomaterials 44 (2015) 173-185.

[16] C. Yang, G. Deng, W. Chen, X. Ye, X. Mo, A novel electrospun-aligned nanoyarnreinforced nanofibrous scaffold for tendon tissue engineering, Colloids Surf. B 122 (2014) 270-276.

[17] V. Kishore, W. Bullock, X. Sun, W.S. Van Dyke, O. Akkus, Tenogenic differentiation of human MSCs induced by the topography of electrochemically aligned collagen threads, Biomaterials 33 (2012) 21372144.

[18] W. Liu, S. Thomopoulos, Y. Xia, Electrospun nanofibers for regenerative medicine, Adv. Healthc. Mater. 1 (2012) 10-25.

[19] B.M. Baker, A.O. Gee, R.B. Metter, A.S. Nathan, R.A. Marklein, J.A. Burdick, R.L. Mauck, The potential to improve cell infiltration in composite fiber-aligned electrospun scaffolds by the selective removal of sacrificial fibers, Biomaterials 29 (2008) 2348-2358.

[20] J. Pu, F. Yuan, S. Li, K. Komvopoulos, Electrospun bilayer fibrous scaffolds for enhanced cell infiltration and vascularization in vivo, Acta Biomater. 13 (2015) $131-141$.

[21] N. Kasoju, U. Bora, Silk fibroin in tissue engineering, Adv. Healthc. Mater. 1 (2012) 393-412.

[22] W.L. Shen, X. Chen, Y.J. Hu, Z. Yin, T. Zhu, J.J. Hu, J.L. Chen, Z.F. Zheng, W. Zhang, J.S. Ran, B.C. Heng, J.F. Ji, W.S. Chen, H.W. Ouyang, Long-term effects of knitted silk-collagen sponge scaffold on anterior cruciate ligament reconstruction and osteoarthritis prevention, Biomaterials 35 (2014) 8154-8163.

[23] J.S. Pieper, A. Oosterhof, P.J. Dijkstra, J.H. Veerkamp, T.H. van Kuppevelt, Preparation and characterization of porous crosslinked collagenous matrices containing bioavailable chondroitin sulphate, Biomaterials 20 (1999).

[24] M. Madaghiele, A. Sannino, I.V. Yannas, M. Spector, Collagen-based matrices with axially oriented pores, J. Biomed. Mater. Res. A 85 (2008) 757-767.

[25] W. Shen, J. Chen, Z. Yin, X. Chen, H. Liu, B.C. Heng, W. Chen, H.W. Ouyang, Allogenous tendon stem/progenitor cells in silk scaffold for functional shoulder repair, Cell Transplant. 21 (2012) 943-958.

[26] P. Chen, J. Tao, S. Zhu, Y. Cai, Q. Mao, D. Yu, J. Dai, H. Ouyang, Radially oriented collagen scaffold with SDF-1 promotes osteochondral repair by facilitating cell homing, Biomaterials 39 (2015) 114-123.

[27] W. Shen, X. Chen, J. Chen, Z. Yin, B.C. Heng, W. Chen, H.W. Ouyang, The effect of incorporation of exogenous stromal cell-derived factor-1 alpha within a knitted silk-collagen sponge scaffold on tendon regeneration, Biomaterials 31 (2010) 7239-7249.

[28] S.A. Grant, C.S. Spradling, D.N. Grant, D.B. Fox, L. Jimenez, D.A. Grant, R.J. Rone, Assessment of the biocompatibility and stability of a gold nanoparticle collagen bioscaffold, J. Biomed. Mater. Res. A 102 (2014) 332-339. 